\title{
HODGE THEORY AND GEOMETRY
}

\author{
PHILLIP GRIFFITHS
}

This expository paper is an expanded version of a talk given at the joint meeting of the Edinburgh and London Mathematical Societies in Edinburgh to celebrate the centenary of the birth of Sir William Hodge. In the talk the emphasis was on the relationship between Hodge theory and geometry, especially the study of algebraic cycles that was of such interest to Hodge. Special attention will be placed on the construction of geometric objects with Hodge-theoretic assumptions. An objective in the talk was to make the following points:

- Formal Hodge theory (to be described below) has seen significant progress and may be said to be harmonious and, with one exception, may be argued to be essentially complete;

- The use of Hodge theory in algebro-geometric questions has become pronounced in recent years. Variational methods have proved particularly effective;

- The construction of geometric objects, such as algebraic cycles or rational equivalences between cycles, has (to the best of my knowledge) not seen significant progress beyond the Lefschetz $(1,1)$

theorem first proved over eighty years ago;

- Aside from the (generalized) Hodge conjecture, the deepest issues in Hodge theory seem to be of an arithmetic-geometric character; here, especially noteworthy are the conjectures of Grothendieck and of Bloch-Beilinson. Moreover, even if one is interested only in the complex geometry of algebraic cycles, in higher codimensions arithmetic aspects necessarily enter. These are reflected geometrically in the infinitesimal structure of the spaces of algebraic cycles, and in the fact that the convergence of formal, iterative constructions seems to involve arithmetic 
as well as Hodge-theoretic considerations. They are also reflected in Hodge-theoretic invariants, based on arithmetic constructions, of cycles in Chow groups.

- The use of non-linear PDE methods, which have proved so powerful in complex geometry, may be applied with considerable effectiveness to Hodge theory;

- The combination of formal homological methods with ideas from the classical topology of algebraic varieties can lead to extensive extensions of the classical Hodge conjecture as well as an attractive proposal for the elusive theory of "motivic cohomology".

In this report we shall use a number of mostly standard abbreviations which we now list:

$\begin{array}{ll}\text { HS } & - \text { Hodge structure } \\ \text { MHS } & - \text { mixed Hodge structure } \\ \text { VHS } & - \text { variation of Hodge structure } \\ \text { HC } & - \text { Hodge conjecture } \\ \text { GHC } & - \text { generalized Hodge conjecture } \\ \text { IVHS } & - \text { infinitesimal variation of Hodge structure } \\ \text { CVHS } & - \text { complex variation of Hodge structure } \\ \text { HYM } & - \text { Hermitian-Yang-Mills } \\ \text { MHC } & - \text { motivic Hodge structure } \\ \text { HCAP } & - \text { Hodge conjecture for admissible pairs } \\ \text { ECM } & - \text { effective cohomological matrices } \\ \text { SCC } & - \text { standard cycle conjectures }\end{array}$

\section{OUtLine}

1. Formal Hodge theory

2. Hodge theory and algebraic cycles

3. Infinitesimal methods in Hodge theory

4. Some arithmetic aspects of Hodge theory

5. Hodge-theoretic invariants of algebraic cycles

6. A use of non-linear PDE in Hodge theory

7. A recent formal construction of Nori

\section{Formal Hodge theory}

The basic objects of formal Hodge theory discussed in this paper are 
- A Hodge-structure (more specifically a polarized Hodge structure of weight $m$ );

- A mixed Hodge structure (again, more specifically a polarized mixed Hodge structure); and

- A variation of Hodge structure and, more generally, a variation of mixed Hodge structure (again one or both with polarizations);

- The period map associated to a variation of Hodge structure.

The first three of these objects are abstractions of respectively the cohomology in degree $m$ of a smooth complex projective algebraic variety, the cohomology of an arbitrary complex algebraic variety, and the cohomology in a family of projective varieties whose general member is smooth (for variation of mixed Hodge structure one drops the assumptions of projectivity and smoothness). The last object - a period map - is essentially a differential geometric formulation of a polarized variation of Hodge structure. We shall not discuss variation of mixed Hodge structure, but instead refer to the references cited below which provide a guide to the literature on this topic.

Formal Hodge theory consists of the study of the above objects and, especially, their interrelationships. Of particular importance for this expository paper are the use of analytic methods to study the algebrogeometric and topological properties of a family of algebraic varieties, both globally and locally when the varieties acquire singularities (and these are of course related). Our emphasis will be on the use of these techniques to construct something of algebro-geometric interest. As there are several existing accounts (cf. [22] for a series of expository papers presenting the state of the subject twenty years ago, [6], [3], [47], and [7] for excellent more comprehensive and recent expositions) of formal Hodge theory, here we shall only summarize the basic definitions and results.

Definition: A Hodge structure of weight $m$ is given by the data $\left(H_{\mathbb{Z}}, F^{p}\right)$ where $H_{\mathbb{Z}}$ is a finitely generated lattice and $F^{p}(p=0, \ldots, m)$ is a decreasing filtration on the complexification $H=H_{\mathbb{Z}} \otimes \mathbb{C}$ such that the maps

$$
F^{p} \oplus \bar{F}^{m-p+1} \rightarrow H
$$

are isomorphisms for all $p$. 
Setting

$$
H^{p, q}=F^{p} \cap \bar{F}^{q}
$$

the condition (1.1) is equivalent to

$$
H=\underset{p+q=m}{\oplus} H^{p, q}, \quad H^{p, q}=\bar{H}^{q, p} .
$$

The relation between them is

$$
F^{p}=\underset{p^{\prime} \geqq p}{\bigoplus} H^{p^{\prime}, m-p^{\prime}} .
$$

We shall use the abbreviation HS for "Hodge structure."

A sub-Hodge structure is given by a sub-lattice $H_{\mathbb{Z}}^{\prime}$ such that, setting $F^{\prime p}=H^{\prime} \cap F^{p},\left(H_{\mathbb{Z}}^{\prime}, F^{\prime} p\right)$ is again a Hodge structure.

Definition: A polarized Hodge structure of weight $m$ is given by the data $\left(H_{\mathbb{Z}}, F^{p}, Q\right)$ where $\left(H_{\mathbb{Z}}, F^{p}\right)$ is a Hodge structure of weight $m$ and

$$
Q: H_{\mathbb{Z}} \otimes H_{\mathbb{Z}} \rightarrow \mathbb{Z}
$$

is a bilinear form satisfying the conditions

$$
\left\{\begin{array}{l}
\text { (i) } Q(u, v)=(-1)^{m} Q(v, u) \\
\text { (ii) } Q\left(F^{p}, F^{m-p+1}\right)=0 \\
\text { (iii) the Hermitian form } Q(C u, v) \text { is definite where } \\
C u=(\sqrt{-1})^{p-q} u, \quad u \in H^{p, q} .
\end{array}\right.
$$

A sub-Hodge structure of a polarized Hodge structure is again a polarized Hodge structure, as is its orthogonal under $Q$, and up to a subgroup of finite index in the lattice the original Hodge structure is a direct sum of the sub-Hodge structure and its orthogonal.

The cohomology groups $H^{m}(X, \mathbb{Z}) /$ torsion of a compact Kähler manifold have a canonical Hodge structure of weight $m$, with (1.2) being the famous Hodge decomposition. In case $X$ is a smooth complex projective algebraic variety with $\omega=c_{1}\left(\mathcal{O}_{X}(L)\right)$ the Kähler class associated to an ample line bundle $L \rightarrow X$, for $m \leqq n=\operatorname{dim} X$ the primitive cohomology (here everything is modulo torsion)

$$
H^{m}(X, \mathbb{Z})_{\text {prim }}=\operatorname{ker}\left\{H^{m}(X, \mathbb{Z}) \stackrel{\omega^{n-m+1}}{\longrightarrow} H^{2 n-m+2}(X, \mathbb{Z})\right\}
$$

has a polarization given by

$$
Q(u, v)=\int_{X} u \wedge v \wedge \omega^{n-m}
$$


Because of the hard Lefschetz theorem (which was first actually proved by Hodge)

$$
H^{m}(X, \mathbb{Q}) \stackrel{\omega^{n-m}}{\sim} H^{2 n-m}(X, \mathbb{Q})
$$

and the primitive decomposition theorem

$$
H^{m}(X, \mathbb{Q})=\bigoplus_{k} \omega^{k} \cdot H^{m-2 k}(X, \mathbb{Q})_{\text {prim }},
$$

the primitive cohomologies are the basic building blocks of the cohomology of a smooth projective variety. In case $L=\mathcal{O}_{X}(1)$ is very ample and $Y \in\left|\mathcal{O}_{X}(1)\right|$ is a smooth hyperplane section, because of the Lefschetz theorems stating that

$$
H^{q}(X, \mathbb{Z}) \rightarrow H^{q}(Y, \mathbb{Z})
$$

is an isomorphism for $q \leqq n-2$ and is injective for $q=n-1$, the cohomology groups $H^{m}(X, \mathbb{Z})$ for $m=n$ and, to a somewhat lesser extent, for $m=n-1$, are of particular importance.

For the definitions of a mixed Hodge structure (MHS) and a variation of Hodge structure (VHS) it will be convenient to work over $\mathbb{Q}$ instead of $\mathbb{Z}$. Thus, e.g. $H_{\mathbb{Q}}$ will denote a finite dimensional $\mathbb{Q}$ vector space and in the geometric case we will have

$$
H_{\mathbb{Q}}=H_{\mathbb{Z}} \otimes \mathbb{Q}
$$

where $H_{\mathbb{Z}}$ is a lattice.

Definition: A mixed Hodge structure is given by data $\left(H_{\mathbb{Q}}, W_{m}, F^{p}\right)$ where $W_{m}$ and $F^{p}$ are a pair of finite filtrations with the weight filtration

$$
\cdots \subset W_{m} \subset W_{m+1} \subset \cdots
$$

defined over $\mathbb{Q}$ and the Hodge filtration

$$
\cdots \subset F^{p} \subset F^{p-1} \subset \cdots
$$

defined on $H=H_{\mathbb{Q}} \otimes \mathbb{C}$, and where the filtrations $F^{p}\left(\mathrm{Gr}_{m}^{W}\right)$ induced by $F^{p}$ on

$$
\mathrm{Gr}_{m}^{W}=W_{m} / W_{m-1}
$$

give a Hodge structure of weight $m$.

There is also the notion of a polarized mixed Hodge structure, whose formal definition we will not give here; the main property is that it gives polarized Hodge structures on the $\mathrm{Gr}_{m}^{W}$ 's. 
The basic example of a mixed Hodge structure is given by the cohomology $H^{k}(X, \mathbb{Q})$ of a complex variety $X$, where the weights satisfy $0 \leqq m \leqq 2 k$. For $X$ smooth but possibly non-complete the weights satisfy $k \leqq m \leqq 2 k$, while for $X$ complete but possibly singular they satisfy $0 \leqq m \leqq k$. These mixed Hodge structures are functorial in all reasonable senses. For a pair of complex algebraic varieties $Y \subset X$ the relative cohomology groups $H^{k}(X, Y)$ have a mixed Hodge structure and the long exact cohomology sequence is an exact sequence of mixed Hodge structures.

Although the definition of a MHS is relatively simple, the underlying linear algebra is rather subtle. For example, a morphism of type $(r, r)$ between MHS's $\left(H_{\mathbb{Q}}, W_{m}, F^{p}\right)$ and $\left(H_{\mathbb{Q}}^{\prime}, W_{l}^{\prime}, F^{\prime q}\right)$ is given by a linear map

$$
L: H_{\mathbb{Q}} \rightarrow H_{\mathbb{Q}}^{\prime}
$$

satisfying

$$
\left\{\begin{array}{l}
L\left(W_{m}\right) \subset W_{m+2 r}^{\prime} \\
L\left(F^{p}\right) \subset F^{\prime p+r} .
\end{array}\right.
$$

Any such morphism is then strict in the sense that $L\left(F^{p}\right)=F^{p+r} \cap \operatorname{Im} L$ and similarly for the weight filtration. Because of strictness, kernels and cokernels of morphisms inherit MHS's.

Definition: A variation of Hodge structure (VHS) is given by data $\left(S, \mathcal{H}_{\mathbb{Q}}, \mathcal{F}^{p}, \nabla\right)$ where

- $S$ is a smooth complex algebraic variety;

- $\mathcal{H}_{\mathbb{Q}}$ is a local system of $\mathbb{Q}$-vector spaces;

- $\mathcal{H}=\mathcal{H}_{\mathbb{Q}} \otimes \mathcal{O}_{X}$ is a holomorphic vector bundle with a filtration $\mathcal{F}^{p} \subset \mathcal{H}$ by holomorphic sub-bundles; and

- $\nabla: \mathcal{H} \rightarrow \Omega_{S}^{1} \otimes \mathcal{H}$ is an integrable connection,

and where the conditions

- $\nabla \mathcal{H}_{\mathbb{Q}}=0$;

- for each $s \in S$, on each fibre the induced data $\left(H_{\mathbb{Q}, s}, F_{s}^{p}\right)$ gives a Hodge structure of weight $m$; and

- the transversality conditions

$$
\nabla \mathcal{F}^{p} \subset \Omega_{S}^{1} \otimes \mathcal{F}^{p-1}
$$

are satisfied. 
The basic example is the cohomology along the fibres $X_{s}=\pi^{-1}(s)$ of a smooth family

$$
X \stackrel{\pi}{\longrightarrow} S
$$

of compact complex manifolds, where $X$ is Kähler and where

$$
\left\{\begin{array}{l}
\mathcal{H}_{\mathbb{Q}}=R_{\pi_{*}}^{m} \mathbb{Q} \\
F_{s}^{p}=F^{p} H^{m}\left(X_{s}, \mathbb{C}\right) .
\end{array}\right.
$$

There is also the notion of a polarized variation of Hodge structure given by the additional data of

$$
\mathcal{Q}: \mathcal{H}_{\mathbb{Q}} \otimes \mathcal{H}_{\mathbb{Q}} \rightarrow \mathbb{Q}
$$

satisfying $\nabla Q=0$ and inducing a polarized Hodge structure on each fibre. In case (1.6) is quasi-projective we then obtain a polarized VHS by taking the primitive cohomology along the fibres.

A rich interaction between MHS's and VHS occurs in the abstraction of a 1-parameter degenerating family

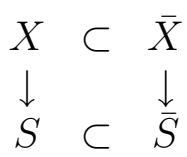

where $\bar{S}=\{|s|<1\}$ is a disc, $S=\bar{S} \backslash\{0\}$ a punctured disc, and $X, \bar{X}$ and $X_{s}$ for $s \neq 0$ are smooth. Then there is a monodromy transformation

$$
T: H_{\mathbb{Z}} \rightarrow H_{\mathbb{Z}}, \quad H_{\mathbb{Z}}=H^{m}\left(X_{s_{0}}, \mathbb{Z}\right) .
$$

It is known (cf. Chapter VI in [22]) that, after possibly passing to a finite covering of $S$, we have

$$
(T-I)^{m+1}=0 .
$$

Setting

$$
N=\log T
$$

a weight filtration is defined on $H_{\mathbb{Q}}$ by the conditions

$$
\left\{\begin{array}{l}
\text { (i) } N: W_{k} \rightarrow W_{k-2} \\
\text { (ii) } N^{k}: W_{m+k} / W_{m+k-2} \stackrel{\sim}{\longrightarrow} W_{m-k} / W_{m-k-2} .
\end{array}\right.
$$


Letting $s=\exp (\sqrt{-1} t)$ where $\operatorname{Im} t>0$, in the early 1970's Schmid proved that

$$
\lim _{\operatorname{Im} t \rightarrow \infty} F_{s}^{p}=: F_{\infty}^{p}
$$

exists and that $\left(H_{\mathbb{Q}}, W_{k}, F_{\infty}^{p}\right)$ gives a polarized MHS relative to which $N$ is a morphism of type $(-1,-1)$ (cf. chapter IV in [22]). The asymptotic analysis leads to extensions $\overline{\mathcal{H}} \rightarrow \bar{S}$ and $\overline{\mathcal{F}}^{p} \rightarrow \bar{S}$ of the Hodge bundles such that

$$
\nabla \overline{\mathcal{H}} \subset \Omega_{\bar{S}}^{1}(\log D) \otimes \overline{\mathcal{H}}, \quad D=\bar{S} \backslash S
$$

In the geometric situation analogous results were proved by Steenbrink (chapter VII in [22]), and a comprehensive understanding of the topology of the pair $X_{s_{0}} \subset X$ was given by Clemens and Schmid (chapter VI in [22]). The extension $\overline{\mathcal{H}}$ is given by $\mathbb{R}_{\pi_{*}}^{m} \Omega_{\bar{X} / \bar{S}}^{\bullet}(\log (\bar{X} \backslash X))$, using the Zariski topology and coherent algebraic sheaves.

There have been numerous applications of the theory. For example,

Let $\left(S, \mathcal{H}_{\mathbb{Q}}, \mathcal{F}^{p}, V\right)$ be a VHS where $S$ is quasi-projective.

Then the mondromy group is semi-simple, and in the geometric case a section of $H^{0}\left(S, \mathcal{H}_{\mathbb{Q}}\right)$ comes by restriction of a class in $H^{m}(\bar{X}, \mathbb{Q})$ (global invariant cycle theorem).

The case of the VHS arising from the abstraction of (1.6) where there are several parameters was finally understood in the mid 1980's through the work of Cattani, Kaplan and Schmid ([8]), Kashiwara [23], Kawamata [24], and by M. Saito [37] in the geometric case. For the study of the asymptotics one may take $\bar{S}$ to be a polycylinder $\left\{\left|s_{i}\right|<1\right\}$ in $\mathbb{C}^{N}$ and

$$
S=\bar{S} \backslash D
$$

where $D$ is the divisor with normal crossings given by $s_{1} \cdots s_{n}=0$. Passing again to finite coverings, the monodromy transformations $T_{i}$ around the punctures $s_{i}=0$ satisfy

$$
\left\{\begin{array}{l}
\left(T_{i}-I\right)^{m+1}=0 \\
{\left[T_{i}, T_{j}\right]=0 .}
\end{array}\right.
$$


A key step in the analysis is the following result of Cattani-Kaplan (cf. chapter $\mathrm{V}$ in $[22]$ )

(1.9) For $\lambda \in\left(\lambda_{1}, \ldots, \lambda_{n}\right) \in \mathbb{R}^{n}$ with $\lambda_{i}>0$, set $N(\lambda)=\sum \lambda_{i} N_{i}$ and let $W(\lambda)_{k}$ be the weight filtration (1.8) defined by $N(\lambda)$. Then $W(\lambda)_{k}$ is independent of $\lambda$.

Roughly speaking, the asymptotic analysis tells us that for each subset $I=\left\{i_{1}, \ldots, i_{k}\right\} \subset\{1, \ldots, n\}$ if we set $D_{I}=\left\{s_{i_{1}}=\cdots=s_{i_{k}}=0\right\}$, then there is over each $D_{I}$ a variation $V_{I}$ of mixed Hodge structure, where the MHS's arise as the Schmid limit as we approach points $s_{0} \in D_{I}$ along discs $\Delta \subset \Delta^{N}$ with $\Delta \cap D=\left\{s_{0}\right\}$. For subsets $I \subset J$ there is a degeneration

$$
V_{I} \rightarrow V_{J}
$$

whose asymptotics also have a precise description. This description depends on an ordering of $\{1, \ldots, n\}$, but geometric conclusions such as those to be described shortly, are independent of the ordering.

An important application of the analysis of the asymptotics of a VHS over a punctured polycylinder as above is the isomorphism, proved by Cattani-Kaplan-Schmid [9] and independenly by Kashiwara [23],

$$
H_{2}^{*}\left(\bar{S}, \mathcal{H}_{\mathbb{Q}}\right) \cong I H^{*}\left(\bar{S}, i_{*} \mathcal{H}_{\mathbb{Q}}\right)
$$

Here, the left-hand side is the $L^{2}$-cohomology with coefficients in the local system $\mathcal{H}_{\mathbb{Q}}$ and where the metrics are the Hodge metrics induced from the polarizations along the fibres and with any metric asymptotic to the Poincaré metric on the base. The right-hand side is the intersection cohomology of the local system $\mathcal{H}_{\mathbb{Q}}$ where $i: S \hookrightarrow \bar{S}$ is the inclusion. A corollary is that

For a VHS over a Zariski open $S$ in a smooth complete variety $\bar{S}$ such that $\bar{S} \backslash S$ is a divisor with normal crossings, $I H^{l}\left(\bar{S}, i_{*} \mathcal{H}_{\mathbb{Q}}\right)$ carries a pure Hodge structure of weight $m+$ $l$.

As will be explained below the appearance of intersection cohomology turns out to be also rooted in geometry, but it seems that the deeper application to geometry of results such as (1.10) may be yet to come.

Another application, due to Cattani-Kaplan-Schmid ([8]), states that 
the currents defined by the Chern forms $c_{k}\left(\Theta_{\bar{F}^{p}}\right)$ of the (singular) Hodge metrics of the extended Hodge bundles are equal to the Chern classes of those bundles

(intuitively, the singularities of $\Theta_{\bar{F}^{p}}$ do not create "residues" along $D$ ).

These results, together with the asymptotic analysis of the VHS, have recently been used to show that

the Kodaira dimension satisfies

$$
\kappa\left(\omega_{\bar{S}}(\log D)\right)=\operatorname{dim} S
$$

if the differential of the period mapping is injective at one point (Zuo [51]), and that the moduli space of canonically polarized complex manifolds is Brody-hyperbolic (Vieweg-Zuo [43]).

In the global geometric case where one has a situation (1.7) with $\bar{X}$ and $\bar{S}$ smooth and projective and the dimension of $\bar{S}$ is arbitrary, the Leray spectral sequence of the map may not degenerate at $E_{2}$ as it does when $\bar{S}$ is a curve. The description of the cohomology of $\bar{X}$ with its Hodge structure in terms of the VHS associated to $X \rightarrow S$ in this case is then replaced by a general structure theorem due to BeilinsonBernstein-Deligne and Gabber [2]. This result essentially describes $H^{*}(\bar{X})$ in terms of the intersection cohomology associated to VHS's over the strata in a stratification of $\bar{S}$. The proof given in [2] uses reduction to characteristic $p$ and the Weil conjectures as proved by Deligne. A proof in the setting of complex algebraic geometry has been given by M. Saito [37] using his theory of mixed Hodge modules. Perhaps because of its technical complexity, the deeper applications of these results to geometry may also be in the future.

Finally recall that a period map is given by a locally liftable, holomorphic mapping

$$
\phi: S \rightarrow \mathcal{D} / \Gamma
$$

where $\mathcal{D}$ is the classifying space for Hodge structures $\left(H_{\mathbb{Z}}, \mathcal{F}^{p}, Q\right)$ with given Hodge numbers $h^{p, q}, \Gamma$ is a subgroup of $\operatorname{Aut}\left(H_{\mathbb{Z}}, Q\right)$ and $\varphi$ satisfies the differential condition

$$
\phi_{*}: T S \rightarrow H T \mathcal{D}
$$


where $H T_{s} \mathcal{D} \subset T_{s} \mathcal{D}$ are the $1^{\text {st }}$ order variations of a Hodge structure $\left(H_{\mathbb{Z}}, F_{s}^{p}, Q\right)$ that satisfy the transversality conditions. An application of the global invariant cycle theorem stated above is the following rigidity result:

A period mapping (1.11) is uniquely determined by its value at one point $s_{0}$ and the induced $\operatorname{map} \phi_{*}: \pi_{1}\left(S, s_{0}\right) \rightarrow$ $\Gamma$.

A long standing question, recently resolved by Kato and Usui ([25]), has been to enlarge $\mathcal{D}$ to a space $\mathcal{D}^{\Sigma}$ of extended Hodge structures with a set of natural properties, the most important being that (1.11) extends to

$$
\bar{\phi}: \bar{S} \rightarrow \mathcal{D}^{\Sigma} / \Gamma
$$

This construction, which is based on the work of Cattani-KaplanSchmid, constructs $\mathcal{D}^{\Sigma} / \Gamma$ as a "fine moduli space for polarized logarithmic Hodge structures with $\Gamma$-level structures". Although not an analytic variety in the usual sense, holomorphic maps to it are welldefined and $\mathcal{D}^{\Sigma} / \Gamma$ does have an infinitesimal structure that allows one to define the differential of the period map at the boundary. As will be illustrated below, this should pave the way for geometric applications of variational techniques to certain singular varieties.

In summary, I believe one may say that formal Hodge theory is now a harmonious subject, and is perhaps one whose main applications to geometry are now begining to reach fruition.

One incomplete aspect of the formal theory regards normal functions. Given a HS of odd weight $m=2 l-1$ there is canonically associated the complex torus

$$
J=H / F^{l}+H_{\mathbb{Z}} .
$$

For a $\operatorname{VHS}\left(S, \mathcal{H}_{\mathbb{Z}}, \mathcal{F}^{p}, \nabla\right)$ of weight $2 l-1$ there is then an associated holomorphic fibre space

$$
\mathrm{J} \rightarrow S
$$

of complex tori. For $\nu$ a holomorphic section of this fibre space, if $v$ is any local lifting to $\mathcal{J}$ then the condition

$$
\nabla v \equiv 0 \quad \bmod \mathcal{F}^{l+1}
$$


is well-defined. Sections $\nu$ of (1.13) satisfying (1.14), together with certain conditions along $\bar{S} \backslash S$ in case $S$ is a quasi-projective algebraic variety, are called normal functions. For geometric reasons to be explained below, a possible missing piece of the formal theory is the extension to $\bar{S}$ of $\mathcal{J} \rightarrow S$ and normal functions $\nu$. As will be explained, here the singularities are "where the action occurs", rather than "loci to be filled in".

A normal function $\nu$ has an important infinitesimal invariant (cf. Chapter XVII in [22], [17], and [44]), denoted by $\delta \nu$, constructed from the intrinsic information in $\nabla v$ and with the property that

$$
\delta \nu=0 \Leftrightarrow \nu \text { has local liftings to } \mathcal{H}_{\mathbb{Z}} \otimes \mathbb{C} .
$$

We will say more below about this invariant.

\section{Hodge theory AND ALGEBRAiC CyCles}

Let $X$ be a smooth, $n$-dimensional complex projective variety. An algebraic cycle

$$
Z=\sum_{i} n_{i} Z_{i}, \quad n_{i} \in \mathbb{Z},
$$

is a finite linear combination of irreducible subvarieties $Z_{i}$ with integer coefficients. Those with codim $Z_{i}=p$ form a group $Z^{p}(X)$. The basic equivalence relation, rational equivalence, is defined by

$$
\sum_{\lambda} \operatorname{div}\left(f_{\lambda}\right) \equiv_{\text {rat }} 0
$$

where $f_{\lambda} \in \mathbb{C}\left(Y_{\lambda}\right)^{*}$ with the $Y_{\lambda}$ being irreducible subvarieties of codimension $p-1$. The quotients

$$
Z^{p}(X) / \equiv_{\mathrm{rat}}=: C H^{p}(X)
$$

are the Chow groups. They have good functorial properties including an intersection pairing

$$
C H^{p}(X) \otimes C H^{q}(X) \rightarrow C H^{p+q}(X) .
$$

The basic Hodge-theoretic invariants of algebraic cycles were already considered by Hodge. They may be expressed by

$$
\begin{array}{ll}
C H^{p}(X) & \stackrel{\psi_{0}}{\longrightarrow} H g^{p}(X)=: H^{2 p}(X, \mathbb{Z}) \cap H^{p, p}(X) \\
\cup & \\
C H^{p}(X)_{\mathrm{hom}} & \stackrel{\psi_{1}}{\longrightarrow} J^{p}(X) .
\end{array}
$$


Here, $\psi_{0}(Z)$ is the fundamental class carried by an algebraic cycle $Z$ and $H g^{p}(X)$ denotes the group of Hodge classes as defined above; $C H^{p}(X)_{\text {hom }}$ is the kernel of $\psi_{0}$. Thinking of an analytic subvariety as defining a current by integration of smooth forms over its regular points, this current is closed and defines $\psi_{0}(Z)$. Hodge noted that

$$
\int_{Z_{i}} \varphi=0, \quad \varphi \in A^{n-p+k, n-p-k}(X) \text { where } k \neq 0
$$

so that $\psi_{0}(Z)$ is a Hodge class. He conjectured that $\psi_{0}$ is onto. Atiyah and Hirzebruch [1] showed that this is false for torsion classes when $p \geqq 2$, and the famous Hodge conjecture ( $\mathrm{HC}$ ) is that $\psi_{0}$ is surjective when everything is tensored with $\mathbb{Q}$. For $p=1$ the $\mathrm{HC}$ is true even with torsion - this is the Lefschetz $(1,1)$ theorem [26].

The intermediate Jacobian

$$
J^{p}(X)=: H^{2 p-1}(X, \mathbb{C}) / F^{p}+H^{2 p-1}(X, \mathbb{Z})
$$

and the Abel-Jacobi map $\psi_{1}$ (sometimes denoted $A J_{X}$ ) is defined on $C H^{p}(X)_{\text {hom }}$ by generalizing the classical procedure for divisors on curves. Using the formalism of currents, we write $Z=\partial \Gamma$ where $\Gamma$ is a real $2 n-2 p+1$ chain and set

$$
\psi_{1}(Z)=\int_{\Gamma} \varphi \quad \bmod \text { periods }
$$

where $\varphi \in F^{n-p+1} H^{2 n-2 p+1}(X, \mathbb{C})$. If $\left\{Z_{t}\right\}_{t \in B}$ is an algebraic family and $\Gamma_{t}$ is the one parameter family of chains with $\partial \Gamma_{t}=Z_{t}$ traced out by a real path in $B$, then again Hodge noted that

$$
\int_{\Gamma_{t}} \psi=0 \text { if } \psi \in A^{n-p+k+1, n-p-k}(X) \text { when } k \neq 0
$$

i.e., algebraic equivalence maps to a sub-Hodge structure in $H^{p+1, p}(X) \oplus$ $H^{p, p+1}(X)$ mod periods.

For $p \geqq 2$ the Abel-Jacobi maps have very non-classical behaviour, viz

- $\operatorname{ker} \psi_{1}$ may be infinite dimensional (Mumford [30])

- $\operatorname{im} \psi_{1}$ may be discrete and non-finitely generated (Clemens [12]). 
Conjectures of Beilinson, building on earlier conjectures of Bloch and ultimately related to the fact that

$$
\operatorname{Ext}^{i}(*, *)=0, \quad i \geqq 2
$$

in the category of MHS's, would establish some order in the situation. Both these conjectures and the $\mathrm{HC}$ involve constructing something geometric - either a rational equivalence or an algebraic cycle — with Hodge theoretic assumptions.

Next, I want to explain some of the geometry behind the missing piece of formal Hodge theory as explained above. ${ }^{1}$ If we have the geometric situation

$$
\begin{aligned}
& X \subset \bar{X} \\
& \pi \downarrow \downarrow \downarrow \\
& S \subset \bar{S} \\
& Z \in Z^{p}(X), \quad Z_{s}=Z \cdot X_{s} \sim 0 \text { for } s \in S
\end{aligned}
$$

then the family of intermediate Jacobians $\left\{J^{p}\left(X_{s}\right)\right\}_{s \in S}$ forms a fibre space $\mathcal{J} \rightarrow S$ of complex tori and

$$
\nu_{Z}(s)=: \psi_{1}\left(Z_{s}\right) \in J\left(X_{s}\right)
$$

gives a normal function. In case $\operatorname{dim} S=1$ and we can write

$$
Z_{s}=\partial \Gamma_{s}
$$

where $\Gamma_{s}$ is invariant as $s$ turns around points of $D=\bar{S} \backslash S$, extending some earlier work El Zein and Zucker (cf. Chapter XV in [22]) showed that we may fill in the family $J^{p}\left(X_{s}\right)$ by using the extended Hodge bundles and the invariant part of the lattice, and then when this is done $\nu_{Z}$ extends to $\bar{S}$. In case (2.3) is not satisfied but the local monodromy satisfies

$$
(T-I)^{2}=0
$$

Clemens ([11]) and M. Saito ([38]) have shown that we may extend $J^{p}\left(X_{s}\right)(s \in D)$ by the finite group

$$
\operatorname{ker}(T-I) / \operatorname{im}(T-I)
$$

\footnotetext{
${ }^{1}$ The following discussion has benefitted from correspondence with Herb Clemens, Mark Green and Richard Thomas.
} 
to obtain a Néron model $\tilde{J}^{p}\left(X_{s}\right)$ and then $\nu_{Z}$ extends to $\nu_{Z}(s) \in$ $\tilde{J}^{p}\left(X_{s}\right)$. However, for geometric reasons to be explained now one should consider the situations (2.1), (2.2) where both $\operatorname{dim} S$ and the singularities of the degenerations are arbitrary.

Because of the Lefschetz theorems and the primitive decomposition, the crucial case of the $\mathrm{HC}$ is when

$$
\left\{\begin{array}{l}
\operatorname{dim} X=2 n \\
\gamma \in H g^{n}(X) \text { is a primitive Hodge class. }
\end{array}\right.
$$

The classical Lefschetz inductive method is to consider a very ample linear system $\left|\mathcal{O}_{X}(l)\right|$. We then arrive at a situation

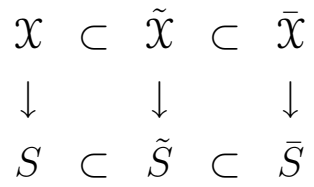

where $\left\{X_{s}\right\}_{s \in S}$ are the smooth hypersurface sections $X_{s} \in\left|\mathcal{O}_{X}(l)\right|$, the $X_{s}$ for $s \in \tilde{S}$ have at most nodal singularities, and $\bar{S}$ is a suitable blow up of $\left|\mathcal{O}_{X}(l)\right|$. A recent result, whose origins go back some thirty years, is (cf. Chapter XIV in [22], [38])

$$
\begin{aligned}
& \text { A Hodge class } \gamma \in H^{n}(X)_{\text {prim }} \text { defines } \\
& \text { an extended normal function } \nu_{\gamma} \text {. }
\end{aligned}
$$

Here extended means that $\nu_{\gamma}$ is defined over $\tilde{S}$ and that $\nu_{\gamma}(s) \in \tilde{J}^{p}\left(X_{s}\right)$ when $X_{s}$ is nodal. Of course, if $\gamma=\psi_{0}(Z)$ for an algebraic cycle $Z$ then $\nu_{\gamma}=\nu_{Z}$ and the extension in (2.7) is the one discussed earlier. Although (2.7) does associate to a Hodge class a global analytic object, it's usefulness has so far been limited by the failure of Jacobi inversion in higher codimension.

Now it has again been known for some time (cf. [21]) that one should in fact focus on the behaviour of normal functions at the singular $X_{s}$ $(s \in D)$, for the following reasons: If $\gamma \in \psi_{0}(Z)$ then by moving $Z$ in a rational equivalence and working modulo torsion we may assume that $Z=\sum_{i} n_{i} Z_{i}$ where the $Z_{i}$ are smooth and in general position. Then for $l \gg 0$, a standard argument shows that there are nodal $X_{s_{0}} \in\left|\mathcal{O}_{X}(l)\right|$ with

$$
|Z|=\bigcup_{i} Z_{i} \subset X_{s_{0}}
$$


If $p_{\lambda}$ are the nodes on $X_{s_{0}}$ with vanishing cycles

$$
\delta_{\lambda} \in H_{2 n-1}\left(X_{s}, \mathbb{Z}\right)
$$

where $\delta_{\lambda} \rightarrow p_{\lambda}$ as $s \rightarrow s_{0}$, then if $\gamma \neq 0$ there must be a non-trivial linear relation

$$
\sum_{\lambda} m_{\lambda} \delta_{\lambda}=0, \quad \quad m_{\lambda} \in \mathbb{Z}
$$

among the vanishing cycles. Moreover, the monodromy around $s_{0}$ satisfies (2.4), the group (2.5) is non-trivial and in general

$$
0 \neq\left[\nu_{Z}\left(s_{0}\right)\right] \in \tilde{J}^{n}\left(X_{s_{0}}\right) / J^{n}\left(X_{s_{0}}\right) .
$$

In fact, analysis of Lefschetz's proof of (1.4) shows that for nodal hypersurface sections the condition that the image of

$$
H_{2 n}\left(X_{s_{0}}, \mathbb{Z}\right) \rightarrow H_{2 n}(X, \mathbb{Z})
$$

contain new cycles - i.e., cycles whose primitive component is non-zero - is (2.8). Moreover if there is a primitive algebraic cycle $Z$ supported on $X_{s_{0}}$ then (2.8) is satisfied.

One problem is that the invariant (2.9) is torsion and the property formulated $\mathrm{HC}$ does not see torsion phenomena. Moreover, geometrically one should not restrict to 1-parameter families but rather use the full linear system $\left|\mathcal{O}_{X}(l)\right|$. For example the computable "algebraic part" of the infinitesimal invariant $\delta \nu$ of a normal function is non-trivial only when the parameter space is higher dimensional. In addition, examples show that when one uses a higher dimensional parameter space - but still restricting to only nodal singularities — non-torsion phenomena arise in the singularities of $\nu_{\gamma}$.

It is interesting to note that intersection homology naturally enters in the singularities of $\nu_{\gamma}$. Taking the case where $\gamma=\psi_{0}(Z)$ is the fundamental class of a primitive algebraic cycle which does not pass through a node, in case $X_{s} \in\left|\mathcal{O}_{X}(l)\right|$ is either smooth or has independent nodes we have

$$
Z_{s}=Z \cdot X_{s} \sim 0 \text { in } X_{s} \backslash\{\text { nodes }\},
$$

which using the theory of intersection homology [28] we write as

$$
\left[Z_{s}\right]=0 \text { in } I H_{2 n-2}\left(X_{s}\right) .
$$


However, restricting still to nodal $X_{s}$ one has the result that

$$
\text { sing } \nu_{Z}=\left\{s:\left[Z_{s}\right] \neq 0 \text { in } I H_{2 n-2}\left(X_{s}\right)\right\} \text {. }
$$

All of this suggests that

To the story of formal Hodge theory discussed above

should be added the analysis of intermediate Jacobians

and normal functions for a VHS with an arbitrary base.

Being optimistic, one might hope that when this theory is applied to the geometric situation (2.6), the result (2.7) would generalize to give an extended normal function $\bar{\nu}_{\gamma}$ defined over all of $\bar{S}$. If we take only that part $\tilde{S}$ of $\bar{S}$ corresponding to nodal hypersurface sections, then as discussed above both $\mathcal{J} \rightarrow S$ and $\nu_{\gamma}$ extend to give

$$
\begin{array}{llll}
\mathcal{J} & \subset & \tilde{\jmath} \\
\downarrow & \downarrow & \\
S & \subset & \widetilde{S} \subset \bar{S}
\end{array}
$$

and $\nu_{\gamma} \in \Gamma(\tilde{S}, \tilde{J})$. Moreover, it can be shown that assuming the HC in degrees $\leq n-1$

$$
\gamma \text { is an algebraic class } \Leftrightarrow \operatorname{sing}\left(\nu_{\gamma}\right) \neq 0 \text { for } l \geqq l_{0} \text {. }
$$

However, my feeling is that one needs the full extension of (2.10) to $\bar{\jmath} \rightarrow \bar{S}$ with, as a best case scenario, the properties that

$$
\begin{cases}\text { (i) } & \nu_{\gamma} \text { extends to } \bar{\nu}_{\gamma}: \bar{S} \rightarrow \overline{\mathcal{J}} \\ \text { (ii) } \delta \bar{\nu}_{\gamma} \in H^{0}\left(\Omega_{\bar{S}}^{1}(\log D) \otimes \mathcal{K}\right) \\ \text { (iii) } \operatorname{Res}_{D}\left(\delta \nu_{\gamma}\right) \neq 0 \Leftrightarrow \operatorname{sing} \bar{\nu}_{\gamma} \neq \phi \\ \text { (iv) } \operatorname{sing} \bar{\nu}_{\gamma} \neq \phi \Leftrightarrow \gamma \text { is an algebraic class. }\end{cases}
$$

Moreover, my feeling is also that if (i) is done then (ii), (iii), (iv) may follow using existing technology. The upshot would be that to a Hodge class $\gamma$ there is canonically associated an algebro-geometric object $\delta \bar{\nu}_{\gamma}$ that will have singularities if, and only if, $\gamma$ is an algebraic class.

In fact, although the extension $\bar{\gamma}$ has not been defined, recent correspondence with Mark Green suggests that using the Cattani-Kaplan result (1.9) it is possible to define the mod-torsion part, denoted

$$
\operatorname{sing}(\nu) \otimes \mathbb{Q},
$$

of the singularities of a normal function $\nu$. The issue is local in a polycylindrical neighborhood as above (1.9). Following [9] we define 
the complex

$$
\left\{\begin{array}{l}
B^{\bullet}=\underset{0 \leqq p \leqq n}{\bigoplus} B^{p} \\
B^{p}=\underset{I}{\oplus} N_{I} H_{\mathbb{Q}}
\end{array}\right.
$$

where $I$ runs over index sets $I=\left(i_{1}, \ldots, i_{p}\right)$ with $i_{1}<\cdots<i_{p}$ and the boundary map

$$
B^{p} \rightarrow B^{p+1}
$$

is a Koszul-type operator. It is known that (loc. cit.)

$$
H^{q}\left(B^{\bullet}\right) \cong I H^{q}\left(\mathcal{H}_{\mathbb{Q}}\right)
$$

where $\mathcal{H}_{\mathbb{Q}}$ is the local system over the punctured polycylinder and $I H^{q}\left(\mathcal{H}_{\mathbb{Q}}\right)$ are the associated intersection cohomology groups $([28])$. The proposal referred to is

$$
\operatorname{sing}(\nu) \otimes \mathbb{Q} \cong H^{1}\left(B^{\bullet}\right) .
$$

Here of course the left-hand-side needs to be defined precisely; this can be done and it exactly measures the obstruction to extending some integral multiple of $\nu$ to the identity component of the canonical extension of the family of intermediate Jacobians.

We note that (2.13) is zero when the dimension of the base space is one, which is consistent with the earlier comment that non-torsion singularities of normal functions can occur only when the base is higher dimensional.

The proof of (2.13) uses both the Cattani-Kaplan result (1.9) and the local invariant cycle theorem as a consequence of the Clemens-Schmid exact sequence (cf. Chapter VI in [22]).

This would be a very nice geometric story. However, we emphasize that at present there are certainly no ideas about how to show that $\delta \bar{\nu}_{\gamma}$ must have singularities. In fact, one expects singularities only when $l$ is large enough that some $X_{s} \in\left|\mathcal{O}_{X}(l)\right|$ supports a class $\lambda$ in $H_{2 n}(X, \mathbb{Q})$ with

$$
\lambda \cdot \gamma \neq 0
$$

where we consider $\gamma \in H_{2 n}(X, \mathbb{Q})$ using Poincaré duality. When $X$ is an algebraic surface and we write

$$
\gamma \cdot \gamma=-k^{2}, \quad k>0
$$


then it may be shown that we must have

$$
l \geqq(\text { constant }) k
$$

to expect singularities for $\nu_{\gamma}$. When $n \geqq 2$, even if $\gamma$ is assumed to be the fundamental class of a primitive algebraic cycle no estimate such as (2.14) is known.

The existence of singularities of normal functions for $l \gg 0$ is related to the following geometric question:

Let $\gamma \in H g^{p}(X)$ be the class of an algebraic cycle and $\lambda \in H g^{1}(X)$ the class $c_{1}\left(\mathcal{O}_{X}(1)\right)$. Then it is evident that there exists an $l_{0}$ such that for $l \geqq l_{0}$

$$
\gamma+l \lambda^{p}=\psi_{0}(Z)
$$

where $Z$ is an effective cycle (i.e., a subvariety).

The question is

Can we estimate $l_{0}$ in terms of $\gamma, \lambda$, and the $c_{i}(X)$.?

For $p=1, n-1$ this is true (and one need only assume that $\gamma$ is a Hodge class). For $p=2, n=4$ it does not seem to be known, one way or the other.

\section{Infinitesimal METhods in Hodge THEORY}

The infinitesimal information, denoted by IVHS (cf. Chapter III in $[22]$ ), in the VHS that arises from a family of smooth algebraic varieties has been seen to contain surprisingly rich geometric information (comprehensive accounts are given in [18], [46], and [7]). For example, for the local moduli $\left\{X_{s}\right\}_{s \in S}$ of smooth algebraic curves of genus $g \geqq 2$, the period domain $\mathcal{D}$ is the Siegel generalized upper-half-plane of $g \times g$ symmetric matrices with positive definite imaginary part, and one has natural identifications

$$
\begin{aligned}
& T^{*}(\mathcal{D}) \cong \operatorname{Sym}^{2} H^{0}\left(\Omega_{X_{s_{0}}}^{1}\right) \\
& T_{s_{0}}^{*}(S) \cong H^{0}\left(\Omega_{X_{s_{0}}}^{\otimes 2}\right) .
\end{aligned}
$$

Moveover, the codifferential of the natural map

$$
\text { (local moduli space of curves) } \rightarrow \mathcal{D}
$$


is

$$
\operatorname{Sym}^{2} H^{0}\left(\Omega_{X_{s_{0}}}^{1}\right) \rightarrow H^{0}\left(\Omega_{X_{s_{0}}}^{\otimes 2}\right)
$$

The kernel $K_{s_{0}}$ of (3.1) is identified with the linear system of quadrics through the canonical curve, and for $X_{s_{0}}$ non-hyperelliptic one knows that

$$
\bigcap_{Q \in K_{s_{0}}} Q \cong X_{s_{0}}
$$

so that we may reconstruct $X_{s_{0}}$ from the first order information in its VHS. Of course, in this case the Torelli theorem states that the global period map

$$
\mathcal{M}_{g} \rightarrow\left\{\begin{array}{c}
\text { principally polarized Hodge structures } \\
\text { of weight one with } h^{1,0}=g
\end{array}\right\}
$$

is injective and not just of degree one onto its image, but since for higher weights there is in general no geometric object such as the theta divisor associated to a Hodge structure the classical methods used for the Torelli theorem are not available and - at least to date - their replacement by infinitesimal methods has been a particularly fruitful approach.

For example we consider a smooth hypersurface

$$
X \subset \mathbb{P}^{n+1}, \quad n \geqq 2
$$

defined by a polynomial equation of degree $d \geqq 3$

$$
F\left(x_{0}, \ldots, x_{n+1}\right)=0 \text {. }
$$

Setting

$$
\left\{\begin{array}{l}
\mathbb{C}\left[x_{0}, \ldots, x_{n+1}\right]=\oplus S_{k} \\
J_{F, k}=S_{k} \cap\left\{F_{x_{0}}, \ldots, F_{x_{n+1}}\right\} \\
R_{k}=S_{k} / J_{F, k}
\end{array}\right.
$$

one has in analogy to (3.1) that the first order information in the VHS associated to varying the equation (3.3) is given by the data

$$
R_{N+q d} \otimes R_{d} \rightarrow R_{N+(q+1) d}, \quad N=d-n-2
$$

where $0 \leqq q \leqq n-1$. Here the subtlety is that we are only given the $R_{k}$ as abstract vector spaces, not as quotients of $S_{k}$ - i.e., we are not given the vector space $S_{1}$ and $R_{k}$ as a quotient of $\operatorname{Sym}^{k}\left(S_{1}\right)$. Ron 
Donagi ([22]), with later refinements by Donagi-Green ([18]) and Voisin ([45]) (cf. Chapters XII and XIII in [22]), showed that

Except for the cases $(d, n)=(3,2),(4,4 m),(6,6 m+1)$ or $(m, m k-2)$ the data (3.4) is sufficient to construct the equation (3.3) up to a projective equivalence.

In fact, by a very clever algebraic argument they show that from (3.4) one may first reconstruct the vector space $S_{1}$ and from that the polynomial ring $\mathbb{C}\left[x_{0}, \ldots x_{n+1}\right]$. Next one may determine the Jacobian ideal $J_{F}$, and then from that information easily construct the polynomial $F$ up to a projective equivalence.

The theme of this talk is the relation between Hodge theory and geometry, especially the construction of geometric objects from Hodge theoretic data. Presently we will discuss how such constructions may arise from infinitesimal Hodge theoretic data. A very interesting application of IVHS to the study of algebraic cycles has been given by Voisin ([46], [48]) who showed

(3.6) Let $X$ be a general, non rigid Calabi-Yau threefold. Then the image of the Abel-Jacobi map

$$
A J_{X}: Z^{2}(X)_{\mathrm{hom}} \rightarrow J^{2}(X)
$$

is a countable and non-finitely generated group.

This result is an existence theorem - algebraic 1-cycles are produced on $X$ and shown to have non-trivial Hodge theoretic properties. An earlier result, first proved by Clemens ([12]) and then later by Voisin ([46], [50]) with a different method using IVHS, is

(3.8) For $X \subset \mathbb{P}^{4}$ a general quintic, the image of the Abel-Jacobi map (3.7) is discrete and non-finitely generated.

In [46] Voisin showed that for a general Calabi-Yau the image of (3.7) is non-torsion. Since the argument used to establish the stronger result (3.6) is an extension of the argument used to prove the non-torsion statement, we shall concentrate on that result.

Voisin's arguments for (3.6) and (3.8) use the infinitesimal invariant for normal functions. In outline algebraic 1-cycles on $X$ are produced as follows: 
Assume for simplicity that $X$ is regular with local moduli space $\left\{X_{s}\right\}_{s \in S}$ and with $X=X_{s_{0}}$. Then $S$ is smooth (Bogomolov, Todorov, Tian [42]) with

$$
\begin{aligned}
T_{s_{0}} S & \cong H^{1}\left(\Theta_{X}\right) \\
& \cong H^{1}\left(\Omega_{X}^{2}\right)
\end{aligned}
$$

where the second identification uses a trivialization

$$
\Omega_{X_{s}}^{3} \cong \mathcal{O}_{X_{s}} \text {. }
$$

Using this trivialization, for a sufficiently ample line bundle $\mathcal{O}_{X}(1)$ the smooth surfaces

$$
S \in\left|\mathcal{O}_{X_{s_{0}}}(1)\right|
$$

have normal bundle

$$
N_{S / X_{s_{0}}} \cong \Omega_{S}^{2}
$$

and depend on

$$
\operatorname{dim} T_{s_{0}}(S)+\operatorname{dim} H^{0}\left(N_{S / X_{s_{0}}}\right)=h^{2,1}\left(X_{s_{0}}\right)+h^{2,0}(S)
$$

parameters. On the other hand, the number of conditions that $\gamma \in$ $H^{2}(S, \mathbb{Z})$ be of Hodge type $(1,1)$ is $h^{2,0}(S)$. Denoting by $H g^{1}(S)_{0}$ the set of Hodge classes $\gamma$ on $S$ that satisfy $\gamma \cdot c_{1}\left(\mathcal{O}_{S}(1)\right)=0$, Voisin shows that

(i) the conditions to be of Hodge type $(1,1)$ are independent, so that

$$
\operatorname{dim}\left\{\left(X_{s}, S, \gamma\right): S \in\left|\mathcal{O}_{X_{s}}(1)\right|, \gamma \in H g^{1}(S)_{0}\right\}=\operatorname{dim} S,
$$

and

(ii) the components of the left hand side of this equality are étalé over $S$.

By the Lefschetz $(1,1)$ theorem the Poincaré dual of $\gamma$ is represented by an algebraic 1-cycle $Z_{\gamma}$ on $S$ homologous to zero in $X_{s_{0}}$; i.e.,

$$
Z_{\gamma} \in Z^{2}\left(X_{s_{0}}\right)_{\text {hom }} \text {. }
$$

The argument is then completed by introducing a refinement of the infinitesimal invariant $\delta \nu_{Z_{\gamma}}$ and showing that this is non-zero, which implies that $\nu_{Z_{\gamma}}$ is non-torsion in a general $J^{2}\left(X_{s}\right)$.

In using IVHS arguments in geometric questions non-trivial multiplicative properties of sheaf cohomology groups always arise. Here the 
property is that, for $\mathcal{O}_{X}(1)$ sufficiently ample and after making the identifications

$$
T_{S}\left|\mathcal{O}_{X}(1)\right|=H^{0}\left(N_{S / X}\right) \cong H^{0}\left(\Omega_{S}^{2}\right)
$$

the Gauss-Manin connection for the family $\left|\mathcal{O}_{X}(1)\right|$ at $S$ gives

$$
H^{1}\left(\Omega_{S}^{1}\right) \stackrel{\bar{\nabla}}{\longrightarrow} \operatorname{Hom}\left(H^{0}\left(\Omega_{S}^{2}\right), H^{2}\left(\mathcal{O}_{S}\right)\right) .
$$

The needed multiplicative property, first formulated by Green ([17]), is

There exists $\lambda \in H^{1}\left(\Omega_{S}^{1}\right)_{0}$ such that $\bar{\nabla}(\lambda)$ is an isomorphism in (3.10).

Geometrically, this property translates into the implication that, as $S$ moves in $\left|\mathcal{O}_{X}(1)\right|$ the subspace $H^{1,1}(X)_{\text {prim }} \cap H^{2}(X, \mathbb{R}) \subset H^{2}(S, \mathbb{R})$ moves non-trivially to intersect $H^{2}(S, \mathbb{Q})$, thus producing Hodge classes. This, together with the Lefschetz $(1,1)$ theorem, are the ingredients to construct algebraic cycles in Voisin's result.

Infinitesimal methods have more commonly been used to prove nonexistence results, where they typically seem to be sharp. For example, referring to (3.8), Green ([17]) and Voisin ([44]) independently showed

For a general threefold $X \subset \mathbb{P}^{4}$ of degree $d \geqq 6$, the image of $A J_{X}$ is torsion.

The line of argument is to show that for any normal function $\nu$ defined over an open set of $X$ 's of degree at least six one has

$$
\delta \nu=0
$$

and conclude with a global monodromy argument.

The results (3.6) and (3.5) date back some years; detailed expositions of these and related results are given in [18] and [45] and [7]. More recently variants on the techniques used for them have been applied by Müller-Stach ([29]), Collino ([13]) and others to construct indecomposable cycles in Bloch's higher Chow groups, to mention just one instance of the ongoing use of infinitesimal Hodge theoretic methods in geometric questions.

The idea of their line of argument is this: A cycle in $C H^{2}(X, 1)$ is represented by $Z=\sum_{\lambda}\left(Y_{\lambda}, f_{\lambda}\right)$ where the $Y_{\lambda}$ are irreducible curves, 
$f_{\lambda} \in \mathbb{C}\left(Y_{\lambda}\right)^{*}$ and

$$
\sum_{\lambda} \operatorname{div}\left(f_{\lambda}\right)=0
$$

Let $\gamma_{\lambda}=f_{\lambda}^{-1}(0, \infty)$ and set $\gamma=\sum_{\lambda} \gamma_{\lambda}$. Then $\partial \gamma=0$ and (working modulo torsion) one has

$$
\gamma=\partial \Gamma, \quad \Gamma=2 \text {-chain on } X .
$$

The current

$$
\varphi \rightarrow \sum \int_{X_{\lambda}-\gamma_{\lambda}}\left(\log f_{\lambda}\right) \varphi+2 \pi \sqrt{-1} \int_{\Gamma} \varphi \quad \bmod \text { periods }
$$

gives the regulator $\rho(Z) \in H_{D}^{2}(X, \mathbb{Z}(1))$. One then defines an infinitesimal invariant $\delta \rho$ and uses geometry to show that $\delta \rho \neq 0$ in the relevant families.

Related to the problem stated in section 2 above of extending $\mathcal{J} \rightarrow S$ and $\nu$ to $\bar{S}$ is to extend the IVHS techniques to the boundary $D$ and, especially, to draw geometric conclusions from this. In this regard, based on the Kato-Usui extension of period maps discussed above, recent work of S. Saito ([39]) has extended the results of Donagi et al. to certain singular hypersurfaces, thereby providing a step in this direction.

Finally, one interpretation of the $\delta \nu$ 's arises from the fundamental class $\psi_{0}(Z)$ of an algebraic cycle $Z$ expressed in the Leray spectral sequence for $\mathbb{R}^{2 p} \Omega_{X / S}^{\bullet}$ for a smooth family $X \rightarrow S$ where $S$ is affine. To say that $\psi_{0}\left(Z_{s}\right)=0$ means that $\psi_{0}(Z)$ lives in the first filtration level $F^{1}$ for that spectral sequence, and $\delta \nu_{Z}$ is essentially the $G r^{1}=F^{1} / F^{2}$ term. If this is zero one may go on to define higher $\delta^{k} \nu_{Z}$ 's. From this perspective a far reaching result of Nori ([34]) with subsequent refinements by a number of people — especially Nagel ([32]) — leads among other things to results of the type (3.11) in situations where there is no classical intermediate Jacobian. An excellent account of this development is given in [7].

\section{Some ARIthmetic Aspects of Hodge theory}

One of the first realizations that, even if one is only interested in the complex geometry of algebraic varieties, arithmetic aspects necessarily 
enter in higher codimension was in Spencer Bloch's formula

$$
T_{f} C H^{2}(X) \cong H^{2}\left(\Omega_{X / \mathbb{Q}}^{1}\right)
$$

for the formal tangent space $T_{f} C H^{2}(X)$ of the Chow group of 0-cycles on a complex algebraic surface $X$. On the right hand side of (4.1) everything is in the algebraic setting - cohomology is in the Zariski topology, and $\Omega_{X / \mathbb{Q}}^{1}$ is the sheaf of absolute Kähler differentials associated to the structure sheaf $\mathcal{O}_{X}$ whose stalks $\mathcal{O}_{X, x}$ are the regular, rational functions in a Zariski neighborhood of $x \in X$.

The origin of (4.1) is two-fold. The first is Bloch's identification

$$
C H^{2}(X) \cong H^{2}\left(\mathcal{K}_{2}\left(\mathcal{O}_{X}\right)\right)
$$

of the Chow group of 0 -cycles with the cohomology of the sheaf $\mathcal{K}_{2}\left(\mathcal{O}_{X}\right)$ with stalks

$$
\mathcal{K}_{2}\left(\mathcal{O}_{X}\right)_{x}=K_{2}\left(\mathcal{O}_{X, x}\right)
$$

arising from the higher $K$-groups of a ring, and the second is van der Kallen's identification of the formal tangent space

$$
T_{f} K_{2}(R) \cong \Omega_{R / \mathbb{Q}}^{1}
$$

where $R$ is a commutative ring containing $\mathbb{Q}$ and $\Omega_{R / \mathbb{Q}}^{1}$ are the absolute Kähler differentials, defined as the $R$-module generated by symbols

$$
d r, \quad r \in R
$$

subject to the relations

$$
\left\{\begin{array}{l}
d\left(r+r^{\prime}\right)=d r+d r^{\prime} \\
d\left(r r^{\prime}\right)=r d r^{\prime}+r^{\prime} d r .
\end{array}\right.
$$

The formal tangent space is defined as

$$
T_{f} K_{2}(R)=\operatorname{ker}\left\{K_{2}(R[\epsilon]) \rightarrow K_{2}(R)\right\}
$$

where $\epsilon^{2}=0$ and the map is $r+\epsilon r^{\prime} \rightarrow r$. Taking $R=\mathbb{C}$ it is well known (cf. [14]) that for $a \in \mathbb{C}$

$$
d a=0 \text { in } \Omega_{\mathbb{C} / \mathbb{Q}}^{1} \Leftrightarrow a \in \overline{\mathbb{Q}} .
$$

Thus (4.1) has an unavoidably arithmetic aspect.

Recall that for any ring $R$ with $\mathbb{C} \subset R$ one may define $\Omega_{R / \mathbb{C}}^{1}$ by adding the relation

$$
d(a r)=a d r, \quad a \in \mathbb{C}
$$


to (4.4), and there is an exact sequence

$$
0 \rightarrow \Omega_{\mathbb{C} / \mathbb{Q}}^{1} \otimes_{\mathbb{C}} R \rightarrow \Omega_{R / \mathbb{Q}}^{1} \rightarrow \Omega_{R / \mathbb{C}}^{1} \rightarrow 0
$$

In the algebraic setting one has

$$
\Omega_{\mathcal{O}_{X, x} \mathbb{C}}^{1} \cong \mathcal{O}_{X, x}\left(T^{*} X\right)
$$

where the right-hand side are the regular sections of the cotangent bundle, thereby relating Kähler differentials to geometry. In the analytic setting one may of course define $\Omega_{\mathcal{O}_{X^{a n}, x / \mathbb{C}}}^{1}$, but the analogue of (4.6) is false - the axioms of Kähler differentials do not permit term by term differentiation of a power series.

Returning to the general discussion, using (4.5) in (4.1) we have

$$
\Omega_{\mathbb{C} / \mathbb{Q}}^{1} \otimes H^{2}\left(\mathcal{O}_{X}\right) \rightarrow T_{f} C H^{2}(X) \rightarrow H^{2}\left(\Omega_{X / \mathbb{C}}^{1}\right)
$$

The right hand map is the differential of the map

$$
C H^{2}(X) \rightarrow \operatorname{Alb}(X) \text {. }
$$

Using

$$
H^{2}\left(\mathcal{O}_{X}\right) \cong H^{0}\left(\Omega_{X / \mathbb{C}}^{2}\right)^{*}
$$

the left hand map suggests that higher differentials enter in $C H^{2}(X)$, which was in a different way already known from Mumford's proof that

$$
h^{2,0}(X) \neq 0 \Rightarrow \operatorname{dim} C H^{2}(X)=\infty .
$$

If now $X$ is defined over $\overline{\mathbb{Q}}$ and we replace $\mathbb{C}$ by $\overline{\mathbb{Q}}$ in $(4.1)$ we get

$$
\begin{aligned}
T_{f} C H^{2}(X(\overline{\mathbb{Q}})) & \cong H^{2}\left(\Omega_{X(\overline{\mathbb{Q}}) / \mathbb{Q}}^{1}\right) \\
& \cong H^{2}\left(\Omega_{X(\overline{\mathbb{Q}} / \overline{\mathbb{Q}})}^{1}\right. \\
& \cong T \operatorname{Tlb}(X(\overline{\mathbb{Q}}))
\end{aligned}
$$

where the last step follows by using (4.5) replacing $\mathbb{C}$ by $\overline{\mathbb{Q}}$ and the fact that $\Omega_{\overline{\mathbb{Q}} / \mathbb{Q}}^{1}=0$. This suggests formally that for $X$ defined over a number field $k$, rational equivalence and Albanese equivalence for $Z^{2}(X(\bar{k}))$ should coincide (modulo torsion), a special case of a deep conjecture of Beilinson to be discussed below.

It is of interest to try to understand the geometric significance of (4.1). This means that one wants to define geometrically the tangent space

$$
T Z^{2}(X)
$$


and, setting

$$
T_{g} C H^{2}(X)=T Z^{2}(X) / T Z_{\text {rat }}^{2}(X)
$$

to see if there is an isomorphism

$$
T_{f} C H^{2}(X) \cong T_{g} C H^{2}(X)
$$

As will be explained below, such an isomorphism would imply a geometric existence theorem, albeit at the infinitesimal level. In [19] there is a propossed definition of $T Z^{2}(X)$ (and for $X$ of dimension $n$ a proposed definition of $T Z^{n}(X)$ and $T Z^{1}(X)$ - the full definition of the remaining $T Z^{p}(X)$ 's awaits resolution of non-trivial technical issues) and with this proposed definition it is shown that (4.7) holds. As shall now be briefly explained, both the higher differentials and arithmetic considerations enter.

For $X$ an $n$-dimensional smooth variety, the group $Z^{n}(X)$ is generated by configurations of points

$$
x_{1}+\cdots+x_{d} \in X^{(d)}
$$

on $X$. The infinitesimal structure of such configurations is reflected by the geometry of $X^{(d)}$ near the diagonals, where we recall that $X^{(d)}$ is singular for $n \geqq 2, d \geqq 2$. This infinitesimal structure is in turn measured by regular differential $q$-forms $\varphi_{d}$ on $X^{(d)}$, especially those with the hereditary property

$$
\left.\varphi_{d+1}\right|_{X^{(d)}}=\varphi_{d}
$$

where the inclusion $X^{(d)} \hookrightarrow X^{(d+1)}$ is given by

$$
x_{1}+\cdots+x_{d} \rightarrow x_{0}+x_{1}+\cdots+x_{d}
$$

for a fixed point $x_{0} \in X$. We may think of such collections $\left\{\varphi_{d}\right\}$ as $q$-forms on the infinite symmetric product $X^{(\infty)}=\bigcup_{d} X^{(d)}$, a semigroup whose associated group is $Z^{n}(X)$. Among the collections of forms satisfying (4.8) are the traces

$$
\operatorname{Tr} \varphi\left(x_{1}+\cdots+x_{d}\right)=\varphi\left(x_{1}\right)+\cdots+\varphi\left(x_{d}\right)
$$


of regular forms $\varphi \in \Omega_{X}^{q}$ on $X$. It is then a result proved by Ting Fei $\mathrm{Ng}([33])$ that

(4.9) The forms satisfying (4.8) are generated as an exterior algebra by the traces of $\varphi \in \Omega_{X}^{q}$ where $0 \leqq q \leqq n$. The forms of all degrees are required to generate.

This "explains" why higher degree forms should enter into the definition of $T Z^{n}(X)$.

As a general remark, for any algebraic (or analytic) variety $Y$ and point $y \in Y$, one may define the coherent $\mathcal{O}_{Y, y}$-modules $\Omega_{Y, y}^{q}$ of regular $q$-forms. (For example, $\omega \in \Omega_{Y, y}^{q}$ is given by a regular $q$-form on the smooth points in a Zariski neighborhood of $y$ that pulls up to a regular form on one, and hence any, desingularization.) Of course, if $y$ is a smooth point then $\Omega_{Y, y}^{\bullet}=\underset{q \geqq 1}{\bigoplus} \Omega_{Y, y}^{q}$ is generated by $\Omega_{Y, y}^{1}$. In general, the structure of $\Omega_{Y, y}^{\bullet}$ as an exterior algebra over $\mathcal{O}_{Y, y}$ reflects the singularity structure of $Y$ near $y$. The result (4.9) should be understood in this context - the complete description in terms of generators and relations seems not yet to be known.

As to the appearance of arithmetic considerations, we shall give an example that illustrates the essential point. For this we consider in $\mathbb{C}^{2}$ the $\operatorname{arc} z_{\alpha \beta}(t)$ in configurations of points with defining ideal

$$
\left(x^{2}-\alpha y^{2}, x y-\beta t\right) \quad(\alpha \neq 0) .
$$

Let $F$ be the free group generated by the 0-cycles $z_{\alpha \beta}(t)$, and then let $\sim$ be the equivalence relation on $F$ generated by

(i) $z_{i}(t) \sim \hat{z}_{i}(t)$ for $i=1,2 \Rightarrow z_{1}(t) \pm z_{2}(t) \sim \hat{z}_{1}(t) \pm \hat{z}_{2}(t)$

(ii) $z(t) \sim \hat{z}(t) \Rightarrow z(\lambda t) \sim \hat{z}(\lambda t) \quad \lambda \in \mathbb{C}^{*}$

(iii) $m z(t) \sim m \hat{z}(t) \Rightarrow z(t) \sim \hat{z}(t) \quad m \in \mathbb{Z}, m \neq 0$

(iv) if $z(t)$ and $\hat{z}(t)$ are effective arcs in $F$ that have the same tangent at $t=0$ in the Hilbert scheme, then $z(t) \sim \hat{z}(t)$.

One thinks here of the definition of the tangent space to a manifold by imposing axioms on the arcs through a point. Then by an elementary but intricate calculation (cf. [19]) one may show

(4.10) The map

$$
F / \sim \Omega_{\mathbb{C} / \mathbb{Q}}^{1}
$$


given by

$$
z_{\alpha \beta}(t) \longrightarrow \beta \frac{d \alpha}{\alpha}
$$

is a well defined isomorphism.

In particular, for $\alpha \in \overline{\mathbb{Q}}$

$$
z_{\alpha \beta}(t) \sim z_{1 \beta}(t)
$$

It is an interesting exercise to show directly that, when $\alpha$ is a root of unity, one may pass from $z_{\alpha \beta}(t)$ to $z_{1 \beta}(t)$ by a sequence of the "moves" (i)-(iv).

The result (4.7) has the following geometric corollary:

Let $X$ be a surface defined over $\overline{\mathbb{Q}}, x_{i} \in X(\overline{\mathbb{Q}})$ and $\tau_{i} \in$ $T_{x_{i}} X(\bar{Q})$. Suppose that for $\tau=\sum_{i}\left(x_{i}, \tau_{i}\right) \in T Z^{2}(X(\overline{\mathbb{Q}})$ the infinitesimal Albanese condition

$$
\langle\varphi, \tau\rangle=\sum_{i}\left\langle\varphi\left(x_{i}\right), \tau_{i}\right\rangle=0 \quad \varphi \in H^{0}\left(\Omega_{X(\overline{\mathbb{Q}})}^{1}\right)
$$

is satisfied. Then there exists an arc $\left(Y_{\lambda}(t), f_{\lambda}(t)\right)$ in the space

$$
\left\{\begin{array}{c}
\oplus \underset{\mathbb{Q}}{\oplus}(Y)^{*} \\
\text { irreducible curve }
\end{array}\right.
$$

such that the tangent at $t=0$ to the arc $\sum_{\lambda} \operatorname{div}\left(f_{\lambda}(t)\right)$ in $Z^{2}(X)$ is $\tau$.

This existence result is an infinitesimal version of a special case of the following conjecture of Beilinson. (In what follows, we set $G_{\mathbb{Q}}=G \otimes_{\mathbb{Z}} \mathbb{Q}$ for any Abelian group G.)

(4.12) Let $X$ be a smooth, projective complex algebraic variety. Then there exists a canonical filtration $F^{m} \mathrm{CH}^{p}(X)_{\mathbb{Q}}$ whose graded pieces may be defined Hodge theoretically. If $X$ is defined over a number field $k$, then

$$
F^{2} C H^{p}(X(k))_{\mathbb{Q}}=0 .
$$

A number of candidates for $F^{n} C H^{p}(X)$ have been proposed. In [20] we have proposed one that is defined Hodge theoretically and has the character of providing a "test" for rational equivalence, much as in the 
classical Abel's theorem; this construction will be summarized in the next section. For all of these

$$
\left\{\begin{array}{l}
F^{0} C H^{p}(X)_{\mathbb{Q}}=C H^{p}(X)_{\mathbb{Q}} \\
F^{1} C H^{p}(X)_{\mathbb{Q}}=\operatorname{ker} \psi_{0} \\
F^{2} C H^{p}(X)_{\mathbb{Q}}=\operatorname{ker} \psi_{1} .
\end{array}\right.
$$

Showing that the one described in [20] has the required properties depends on the generalized Hodge conjecture and (4.13).

We conclude by illustrating what sort of considerations might be necessary to "integrate" an infinitesimal result such as (4.11) by a convergent iterative process. The one example of a "surface" with $p_{g} \neq 0$ for which (4.13) is known seems to be the relative variety $\left(\mathbb{P}^{2}, T\right)$ where $T$ is the coordinate axis triangle. Concretely, the result is the following:

In $\mathbb{C}^{*} \times \mathbb{C}^{*}$ let $p_{i}=\left(x_{i}, y_{i}\right)$ be a configuration of points with $x_{i}$, $y_{i} \in \overline{\mathbb{Q}}$ and $n_{i} \in \mathbb{Z}$ integers such that

$$
\begin{cases}\text { (i) } & \sum_{i} n_{i}=0 \\ \text { (ii) } & \prod_{i} x_{i}^{n_{i}}=\prod_{i} y_{i}^{n_{i}}=1 .\end{cases}
$$

Then there exists a configuration of curves $Y_{\lambda} \subset \mathbb{P}^{2}(\overline{\mathbb{Q}})$ and functions $f_{\lambda} \in \overline{\mathbb{Q}}\left(Y_{\lambda}\right)^{*}$ such that

$$
\sum_{\lambda} \operatorname{div}\left(f_{\lambda}\right)=\sum_{i} n_{i}\left(x_{i}, y_{i}\right)
$$

and

$$
f_{\lambda} \mid \mathbb{P}^{2} \cap T=1 .
$$

The last condition defines the rational functions on the relative variety $\left(\mathbb{P}^{2}, T\right)$. The proof of this result consists in first showing that in $\mathbb{C}^{*} \otimes_{\mathbb{Z}} \mathbb{C}^{*}$ one may write

$$
\prod_{i}\left(x_{i} \otimes y_{i}\right)^{n_{i}}=\prod_{\sigma}\left(a_{\sigma} \otimes 1-a_{\sigma}\right)
$$

where $a_{\sigma} \in \overline{\mathbb{Q}}^{*} \backslash\{1\}$, and then from the right-hand side explicitly constructing the curves $Y_{\lambda}$ (actually, lines will suffice) and rational functions $f_{\lambda}$ such that (4.17) and (4.18) are satisfied.

What underlies this is the result, due essentially to Bloch and Suslin, that for any algebraically closed field $k$ of characteristic zero there is a filtration

$$
F^{p} C H^{2}\left(\mathbb{P}^{2}, T(k)\right)
$$


with

$$
\left\{\begin{array}{l}
G r^{0} \cong \mathbb{Z} \\
G r^{1} \cong k^{*} \times k^{*} \\
G r^{2} \cong K_{2}(k)
\end{array}\right.
$$

The maps (i) and (ii) above map to $G r^{0}, G r^{1}$ respectively and are the degree and Albanese maps in the current situation. The new ingredient is $G r^{2}$, which has a highly arithmetic character. We are using Garland's theorem [16] that $K_{2}(k)$ is torsion for $k$ a number field, which extends the result of Bass and Tate that $K_{2}(\mathbb{Q})$ is torsion, a result closely related to quadratic reciprocity.

If we now ask the question

Can the geometric size of the data $\left(Y_{\lambda}, f_{\lambda}\right)$ be estimated in terms of the geometric size of the $\left(x_{i}, y_{i}\right)$ and $n_{i}$ ?

then the answer is no. Here, the geometric size of $z=\sum_{i} n_{i}\left(x_{i}, y_{i}\right)$ means $\sum_{i}\left|n_{i}\right|$, and for $\left(Y_{\lambda}, f_{\lambda}\right)$ it means $\sum_{\lambda} \operatorname{deg} F_{\lambda}$ where $F_{\lambda} \subset Y_{\lambda} \times$ $\mathbb{P}^{1} \subset \mathbb{P}^{2} \times \mathbb{P}^{1}$ is the graph of $f_{\lambda}$. In addition to the geometric size, the arithmetic complexity of $z$ - meaning essentially the heights of the $x_{i}$ and $y_{i}$ - must enter in bounding the geometric size of the $\left(Y_{\lambda}, f_{\lambda}\right)$. Some subtle interplay between Hodge theory, geometry and arithmetic seems to be going on here. In particular, examples show that for Hodgetheoretic reasons a convergent iterative process is not always possible when a formal one exists, and it seems that a convergent iterative construction will at each stage require bounds of both geometric and arithmetic complexity.

\section{Hodge-THEORETIC INVARIANTS OF ALGEBRAIC CYCLES}

In this section we shall summarize the construction ([20]) of how, if one assumes the GHC and the conjecture (4.12) of Beilinson, a complete set of Hodge-theoretic invariants mod torsion of a rational equivalence class of algebraic cycles may be given.

Let $X$ be a smooth projective variety, which for simplicity of exposition we assume to be defined over $\overline{\mathbb{Q}}$. Let $Z \in Z^{p}(X)$ be an algebraic cycle defined over a field $k$ which is finitely generated over $\mathbb{Q}$, and let $S$ be a smooth projective variety defined over $\mathbb{Q}$ and with

$$
\mathbb{Q}(S) \cong k
$$


Below we shall describe how one may define the spread

$$
z \in Z^{p}(X \times S)
$$

of $Z$; both $S$ and $Z$ are defined up to ambiguities arising from the different geometric realizations of function field constructions. We shall discuss Hodge-theoretic invariants

$$
\Psi_{0}(Z), \Psi_{1}(Z), \ldots, \Psi_{p}(Z)
$$

with the properties:

(i) $\Psi_{0}(Z)=\Psi_{1}(Z)=0$ if, and only if, $\psi_{0}(Z)=\psi_{1}(Z)=0$,

(ii) assuming the GHC, the invariants (5.2) do not depend on the particular model $S$ satisfying (5.1) and the choice of spread $Z \in$ $Z^{p}(X \times S)$

(iii) still assuming the $G H C$, the invariants (5.2) depend only on the rational equivalence class $[Z] \in C H^{p}(X)_{\mathbb{Q}}$, and the conditions

$$
\Psi_{0}(Z)=\Psi_{1}(Z)=\cdots=\Psi_{p}(Z)=0
$$

do not depend on the particular field $k$; and

(iv) assuming the GHC and the conjecture (4.12) of Beilinson, we have that

(5.3) is satisfied if, and only if, $[Z]=0$ in $C H^{p}(X)$.

These conditions have the following

(5.5) Corollary: Every cycle class $[Z] \in F^{m} C H^{p}(X)_{\mathbb{Q}}$ is a sum of cycle classes defined over fields of tr deg $\leqq m$. We need to use $\operatorname{tr} \operatorname{deg} m$ if, and only if, $\Psi_{m}(Z) \neq 0$.

This shows very clearly how arithmetic considerations necessarily appear in the complex geometry of algebraic cycles in higher codimension.

We shall actually (i) define Hodge-theoretic objects $\mathcal{H}_{m}$ with $0 \leqq$ $m \leqq 2 p$, together with subspaces $\mathcal{K}_{m} \subset C H^{p}(X)_{\mathbb{Q}}$, and (ii) inductively define maps

$$
\varphi_{m}: \mathcal{K}_{m} \rightarrow \mathcal{H}_{m}
$$


such that $\varphi_{m}$ is defined if $\varphi_{0}=\cdots=\varphi_{m-1}=0$ and where

$$
\begin{aligned}
& \operatorname{ker}\left\{\varphi_{0}, \ldots, \varphi_{2 m-2}\right\}=: F^{m} C H^{p}(X)_{\mathbb{Q}}, \quad m \geqq 1 \\
& \| \\
& \operatorname{ker}\left\{\Psi_{0}, \ldots, \Psi_{m}\right\} .
\end{aligned}
$$

We refer to [20] for further discussion about the definition of the $\Psi_{m}$ from $\varphi_{0}, \ldots, \varphi_{2 p}$ and various extension data.

The relation between geometry and arithmetic enters through the aforementioned concept of a spread. Informally, we may think of any complex algebraic variety $Y$ as given by polynomial equations

$$
F_{\lambda}(a, x)=\sum_{I} a_{\lambda I} x^{I}=0
$$

where the coefficients $a_{\lambda I}$ generate a field $k \subset \mathbb{C}$. Since $k$ is finitely generated over $\mathbb{Q}$, the coefficients will satisfy a generating set of equations

$$
G_{i}(a)=0
$$

where $G_{i}(\alpha) \in \mathbb{Q}\left[\ldots, \alpha_{\lambda I}, \ldots\right]$. The spread $y$ of $Y$ is then given by the equations

$$
\left\{\begin{array}{l}
F_{\lambda}(b, x)=\sum_{I} b_{\lambda I} x^{I}=0 \\
G_{i}(b)=0
\end{array}\right.
$$

Letting $S$ be a (smooth, projective) model of the variety defined by the second set of equations, (5.1) is satisfied and there is an obvious map

$$
y \rightarrow S,
$$

for which the fibre over the generic point $a \in S$ is $Y$. The diagram (5.7) is well defined up to a birational mapping defined over $\overline{\mathbb{Q}}$.

The spread construction may also be applied to the cycle $Z \in Z^{p}(X(k))$ to give a cycle

$$
z \in Z^{p}((X \times S)(\overline{\mathbb{Q}})) .
$$

As noted above, both $S$ and $z$ are only defined up to certain ambiguities. The Hodge-theoretic objects $\mathcal{H}_{m}$ and maps (5.6) will be constructed from the fundamental class and Abel-Jacobi maps on $X \times S$.

Specifically, using the Künneth decomposition for the Hodge structures on $H^{2 p}(X \times S)$ and $H^{2 p-1}(X \times S)$, we may decompose the fundamental class and Abel-Jacobi maps into Künneth components and 
the terms up to the middle may schematically be arranged as follows

$$
\begin{array}{llll}
(2 p, 0) & (2 p-1,1) & \cdots & (p, p) \\
& (2 p-1,0) & \cdots & (p, p-1) .
\end{array}
$$

The top row corresponds to the fundamental class and the bottom row to the Abel-Jacobi map; the $(k, l)$ entries correspond to $H^{k}(X) \otimes H^{l}(S)$ in the Künneth decomposition of $H^{k+l}(X \times S)$. The maps (5.6) may be pictured as

$$
\begin{array}{ccccc}
\varphi_{0} & \varphi_{1} & \varphi_{3} & \cdots & \varphi_{2 p-1} \\
& \varphi_{2} & \varphi_{4} & \cdots & \varphi_{2 p} .
\end{array}
$$

Now usually the Abel-Jacobi map is only defined on the kernel of the fundamental class mapping. However, in the current situation an interesting refinement of this is possible; the basic observation is

$$
\begin{aligned}
& \text { The component } \varphi_{2 m} \text { of the Abel-Jacobi mapping on cycles in } \\
& Z^{p}(X \times S) \text { is well-defined on cycles where } \varphi_{0}=\cdots=\varphi_{2 m-1}
\end{aligned}
$$
$=0$.

Moreover, assuming the GHC and using the Lefschetz theorems it may be proved that

Under the birational transformations arising from using a different model for a smooth projective variety $S$ satisfying (5.1) and for the spread $Z \in Z^{p}((X \times S)(\mathbb{Q}))$ of a cycle $Z \in Z^{p}(X(k))$, the invariants $\varphi_{0}, \ldots, \varphi_{2 p}$ correspond.

The argument uses in an essential way that we are working with spreads.

Finally, assuming the GHC and the Beilinson conjecture (4.12) it may be shown that

$$
\text { If } \varphi_{0}(Z)=\cdots=\varphi_{2 p}(Z)=0 \text {, then }[Z]=0 \text { in } C H^{p}(X)_{\mathbb{Q}} \text {. }
$$

The point is that if $\varphi_{0}(Z)=\cdots=\varphi_{2 p}(Z)=0$, then using the GHC we may choose a spread model so that

$$
\psi_{0}(z)=\psi_{1}(z)=0
$$

on $X \times S$. Then the Beilinson conjecture gives that, modulo torsion,

$$
z \equiv{ }_{\text {rat }} 0 \text {. }
$$


The first interesting non-classical case is that of zero cycles on an algebraic surface $X$. Then the spread is given by $\left\{Z_{s}\right\}_{s \in S}$ where for $s \in S$ a general point $Z_{s}$ is a 0 -cycle on $X$ with $Z_{s_{0}}=Z$ and the first invariant

$$
\varphi_{0}(Z) \in H^{4}(X) \otimes H^{0}(S) \cong H^{4}(S)
$$

is given by $\operatorname{deg} Z$. The next invariant

$$
\varphi_{1}(Z) \in H^{3}(X) \otimes H^{1}(S) \cong \operatorname{Hom}\left(H_{1}(S), H_{1}(X)\right) .
$$

If $\varphi_{0}(Z)=\operatorname{deg} Z=0$, then there is an induced mapping

$$
S \rightarrow \operatorname{Alb}(X)
$$

given by $s \rightarrow \operatorname{Alb}\left(Z_{s}-Z_{s_{0}}\right)$ and $\varphi_{1}(Z)$ is corresponding mapping on the first homology. If $\varphi_{0}(Z)=\varphi_{1}(Z)=0$, then the mapping (5.13) is constant and we may set

$$
\varphi_{2}(Z)=\operatorname{Alb}\left(Z_{s}\right)
$$

for any $s \in S$ (this is a special case of (5.10)).

Turning to

$$
\varphi_{3}(Z) \in H^{2}(X) \otimes H^{2}(S) \cap\left(H g^{1}(X) \otimes H g^{1}(S)\right)^{\perp}
$$

where the ()$^{\perp}$ term results from factoring out the part resulting from the ambiguities, we see that

$$
\varphi_{3}(Z) \in \operatorname{Hom}\left(H^{0}\left(\Omega_{X / \mathbb{C}}^{2}\right), H^{0}\left(\Omega_{S / \mathbb{C}}^{2}\right)\right),
$$

and it is easy to verify that for $\omega \in H^{0}\left(\Omega_{X / \mathbb{C}}^{2}\right)$

$$
\varphi_{3}(Z)(\omega)=\operatorname{Tr}_{z} \omega \text {. }
$$

This is the invariant originally used by Mumford [30].

The final invariant

$$
\varphi_{4}(Z) \in H^{2}(X) \otimes H^{1}(S) / F^{2}\left(H^{2}(S) \otimes H^{1}(S)\right)+\text { "integral" }
$$

where "integral" is $H^{3}(X \times S, \mathbb{Z}) \cap\left(H^{2}(X) \otimes H^{1}(S)\right)$. The numerator has a component

$$
\varphi_{4}(Z)^{\prime} \in H^{0,2}(X) \otimes H^{1}(S) \cong\left(H_{1}(S) \otimes H^{0}\left(\Omega_{X / \mathbb{C}}^{2}\right)\right)^{*}
$$

with the following interpretation: Let $\lambda$ be a closed loop in $S$ representing a class $[\lambda] \in H_{1}(S, Z)$ and $\omega \in H^{0}\left(\Omega_{X / \mathbb{C}}^{2}\right)$. For $s \in \lambda$ we have the 0-cycle $Z_{s}$ and 1-chain $\gamma_{s}$ with

$$
\partial \gamma_{s}=Z_{s}
$$


We set

$$
\Gamma=\bigcup_{s \in \lambda} \gamma_{s}
$$

and define

$$
\left\langle\varphi_{4}^{\prime}(Z), \lambda \otimes \omega\right\rangle=\int_{\Gamma} \omega \bmod \text { periods. }
$$

If we have

$$
\gamma_{s}^{\prime}=\gamma_{s}+\delta_{s}, \quad \partial \delta_{s}=0
$$

then, setting $\Delta=\bigcup_{s \in \lambda} \delta_{s}$, with the obvious notation we have

$$
\begin{aligned}
\int_{\Gamma^{\prime}} \omega & =\int_{\Gamma} \omega+\int_{\Delta} \omega \\
& \equiv \int_{\Gamma} \omega \bmod \text { periods. }
\end{aligned}
$$

If

$$
\lambda^{\prime}=\lambda+\partial \Lambda
$$

for some 2-chain $\Lambda$ in $S$, then setting

$$
\begin{aligned}
z_{\Lambda} & =\bigcup_{s \in \Lambda} Z_{s} \\
\Sigma & =\bigcup_{s \in \Lambda} \gamma_{s}
\end{aligned}
$$

we have, again with the obvious notation,

$$
\partial \Sigma=\Gamma^{\prime}-\Gamma-z_{\Lambda}
$$

and then

$$
\begin{aligned}
\int_{\Gamma^{\prime}} \omega & =\int_{\Gamma} \omega+\int_{z_{\Lambda}} \omega \\
& =\int_{\Gamma} \omega
\end{aligned}
$$

since

$$
\int_{z_{\Lambda}} \omega=\int_{\Lambda} \operatorname{Tr}_{z} \omega=0
$$

because $\operatorname{Tr}_{z \omega}=0$.

In conclusion, one might say that for a surface $X$ defined over $\mathbb{Q}$ if we assume the GHC and Beilinson conjectures then the analogue of Abel's theorem and its converse may be summarized in the following 
inductive set of "tests" that a 0 -cycle $Z$ be rationally equivalent to zero:

$$
\begin{array}{cc}
\operatorname{deg} Z=\int_{Z} 1=0, & 1 \in H^{0}\left(\Omega_{X}^{0}\right) \\
\operatorname{Alb}(Z)=\int_{\gamma} \varphi \equiv 0 \text { periods } & \varphi \in H^{0}\left(\Omega_{X}^{1}\right)
\end{array}
$$

where if (i) is satisfied there is a 1-chain $\gamma$ with $\partial \gamma=Z$, and

$$
\left\{\begin{array}{l}
\text { (a) } \operatorname{Tr}_{z} \omega=0 \\
\text { (b) } \int_{\Gamma} \omega \equiv 0 \text { periods }
\end{array}\right\} \quad \omega \in H^{0}\left(\Omega_{X}^{2}\right)
$$

where $Z$ is the spread of $Z$ and, assuming that (ii) and (iii)(a) are satisfied, $\Gamma$ is the 2-chain traced out over a 1-cycle $\lambda$ by the 1-chains $\gamma_{s}$ with $\partial \gamma_{s}=Z_{s}, s \in \lambda$. We may think of this test as given by integrating the $q$-forms in $H^{0}\left(\Omega_{X}^{q}\right), 0 \leqq q \leqq 2$, where at each stage the path of integration can be defined because the previous steps were zero.

\section{A use of NON-Linear PDE in Hodge theory}

Thus far the emphasis in this survey article has been the relationship between Hodge theory and algebraic cycles, especially the construction of cycles satisfying Hodge theoretic conditions. The considerations we have employed have been formal Hodge theoretic and algebro-geometric. In this section we shall give an example of the use of analysis to construct something geometric. It is interesting to note that ultimately what is desired to construct are solutions to an overdetermined PDE system arising from the $\bar{\partial}$-operator. What is actually solved are solutions to a determined PDE system, a system that is implied by the overdetermined one. Then global topological assumptions on the Chern classes imply that the solution to the determined system satisfies the overdetermined one as well. There seems to be no technique for generally solving overdetermined PDE systems, even locally for elliptic linear equations.

One may of course ask if analytic methods may be used to construct algebraic cycles, or holomorphic bundles and/or coherent sheaves, extending the Kodaira-Spencer proof of the Lefschetz $(1,1)$ theorem. I feel that the recent work of Voisin [48] casts some doubt on the efficacy of this approach. 
Before turning to the paricular result to be discussed, it is perhaps worth noting that the title Harmonic Integrals of Hodge's book reflects his introduction of linear PDE concepts into the study of the cohomology of manifolds. Subsequently, Kodaira and Spencer among others extensively applied linear PDE methods to algebraic geometry, in particular giving the above mentioned proof of the Lefschetz $(1,1)$ theorem where the existence of cycles is established by analytic methods. Non-linear PDE methods came to the fore after Yau's proof of the Calabi conjecture. Subsequently, there followed the proofs by Donaldson (for projective varieties) and by Uhlenbeck-Yau (for general compact Kähler manifolds) of the existence of Hermitian-Einstein metrics on stable holomorphic vector bundles over compact Kähler manifolds. These ideas were extended by Carlos Simpson to complex variations of Hodge structure (defined below), a consequence of whose work gives the following existence result [40], [41]: 
(6.1) Any linear representation of the fundamental group of a compact Kähler manifold can be continuously deformed into the monodromy representation of a complex variation of Hodge structure.

Corollary: For $n \geqq 3$, no subgroup of finite index in $\mathrm{SL}_{n}(\mathbb{Z})$ is the fundamental group of a smooth projective variety.

It is this development that we shall shortly give a brief description of.

Before doing this we would like to call attention to the related works on harmonic maps to the period domains (cf. the excellent exposition in chapter 14 of [7] and the references cited therein). A consequence of that work is the result

(6.2) no compact quotient of a non-Hermitian symmetric period domain has the homotopy type of a compact Kähler manifold.

Turning to Simpson's work, one approach is to begin by noticing the somewhat subtle interplay between the flat connection $\nabla$ and metric connection $D$ in a polarized VHS. Here, the metric connection is obtained by writing

$$
\mathcal{H}=\underset{p+q=m}{\oplus} \mathcal{H}^{p, q}
$$

and taking $(-1)^{p}$ times the Hodge metric in the individual $\mathcal{H}^{p, q}$ 's. From the properties of a VHS we may write

$$
\left.\begin{array}{l}
\nabla=\nabla^{\prime}+\nabla^{\prime \prime} \\
D=D^{\prime}+D^{\prime \prime}
\end{array}\right\} \quad \text { type decomposition }
$$

and

$$
\nabla=D+\theta+\theta^{*}
$$

where by $(1.5)$

$$
\theta: \mathcal{H}^{p, q} \rightarrow A^{1,0}\left(\mathcal{H}^{p-1, q+1}\right)
$$

and $\theta^{*}$ is the Hermitian adjoint of $\theta$. The integrability condition $\nabla^{2}=0$ implies

$$
\left\{\begin{array}{l}
\text { (i) } \quad \theta: \mathcal{H}^{p, q} \rightarrow \Omega_{S}^{1}\left(\mathcal{H}^{p-1, q+1}\right) \text { is holomorphic } \\
\text { (ii) } \theta \wedge \theta=0 \\
\text { (iii) the curvature of } D \text { is expressed as } \\
\quad \Theta=\left(-\theta \wedge \theta^{*}+\theta^{*} \wedge \theta\right)
\end{array}\right.
$$

This leads to Simpson's basic 
Definition: A Higgs bundle is given by a pair $(\mathcal{E}, \theta)$ where $\mathcal{E}$ is a holomorphic vector bundle and

$$
\theta: \mathcal{E} \rightarrow \Omega_{S}^{1}(\mathcal{E})
$$

is a holomorphic section of $\Omega_{S}^{1}(\operatorname{Hom}(\mathcal{E}, \mathcal{E}))$ that satisfies

$$
\theta \wedge \theta=0 \text {. }
$$

Working with $C^{\infty}$ sections, a Higgs bundle is given by an operator

$$
\nabla^{\prime \prime}=D^{\prime \prime}+\theta
$$

where

$$
\left\{\begin{aligned}
D^{\prime \prime}: & \mathcal{E} \rightarrow A^{0,1}(\mathcal{E}) \\
\theta: & \mathcal{E} \rightarrow A^{1,0}(\mathcal{E})
\end{aligned} \quad \text { is a }(0,1)\right. \text { connection }
$$

and the integrability condition

$$
\left(\nabla^{\prime \prime}\right)^{2}=0
$$

is satisfied. By type considerations this gives the conclusions (6.4):

$$
\left\{\begin{array}{l}
\left(D^{\prime \prime}\right)^{2}=0 \Rightarrow D^{\prime \prime} \text { gives a complex structure on } \mathcal{E} \\
D^{\prime \prime} \theta+\theta D^{\prime \prime}=0 \Rightarrow \theta \text { is holomorphic; and } \\
\theta \wedge \theta=0
\end{array}\right.
$$

Definition: A complex variation of Hodge structure (CVHS) is given by holomorphic Hermitian vector bundles $\mathcal{E}^{p, q}$ with metric connections $D^{p}$ and maps

$$
\theta^{p}: \mathcal{E}^{p, q} \rightarrow A^{1,0}\left(\mathcal{E}^{p-1, q+1}\right)
$$

such that for $D=\underset{p}{\oplus^{p}} D^{p}$ and $\theta=\underset{p}{\oplus_{p}} \theta^{p}$, setting

$$
\nabla=D+\theta+\theta^{*}
$$

the integrability condition

$$
\nabla^{2}=0
$$

is satisfied.

Thus a CVHS is the same as a polarized VHS dropping the real structure in the fibres. In particular, to a CVHS is associated a representation of $\pi_{1}(S)$ into a group of Hodge type. Clearly a CVHS gives a Higgs bundle by taking

$$
\left\{\begin{aligned}
\mathcal{E} & =\oplus \mathcal{E}^{p, q} \\
\nabla^{\prime \prime} & =D^{\prime \prime}+\theta .
\end{aligned}\right.
$$


Next we observe that, essentially because of the expression (iii) in (6.4) for the curvature of a CVHS and the general principal that "curvatures decrease on holomorphic sub-bundles", the Higgs bundle associated to a CVHS has the important property of being semistable. (An account of the curvature properties of Hodge bundles and period domains is given in [7].) In general, we recall that over an $n$-dimensional compact Kähler manifold $S$ with Kähler form $\omega$, we may define the degree of any coherent sheaf $\mathcal{F}$ by

$$
\operatorname{deg} \mathcal{F}=\int_{S} c_{1}(\mathcal{F}) \wedge \omega^{n-1} .
$$

A holomorphic vector bundle $\mathcal{E}$ of rank $r$ is then said to be stable, relative to the Kähler class $[\omega] \in H^{2}(S, \mathbb{R})$, if for any proper coherent subsheaf $\mathcal{F} \subset \mathcal{E}$ the inequality

$$
\frac{\operatorname{deg}(\mathcal{F})}{\operatorname{rk}(\mathcal{F})}<\frac{\operatorname{deg}(\mathcal{E})}{\operatorname{rk}(\mathcal{E})}
$$

holds. Semi-stability is the same condition with $\leqq$ replacing $<$. The basic result, due to Donaldson and Uhlenbeck-Yau, is that

a stable bundle has a unique HYM metric.

This means the following: Any metric in $\mathcal{E}$ gives a canonical metric connection $D$ with curvature $\Theta=D^{2}$. The metric is said to be Hermitian-Yang-Mills (HYM) in case

$$
\Lambda \Theta=\lambda I
$$

where $\Lambda$ is the usual Kähler operator associated to $\omega$ and $I$ is the identity.

The Chern forms associated to a HYM metric satisfy the pointwise inequality

$$
\left(c_{2}-\frac{r-1}{2 r} c_{1}^{2}\right) \wedge \omega^{n-2} \geqq 0
$$

which implies the inequality of Bogomolov for stable bundles. (Here, one recalls Hodge's proof of his index theorem.) Moreover, from this and related formulas one may conclude that if globally we assume that

$$
\int_{S} c_{1} \wedge \omega^{n-1}=0, \quad \int_{S}\left(c_{2}-\frac{c_{1}^{2}}{2}\right) \wedge \omega^{n-2}=0
$$


then suitable pointwise integrands must vanish, implying that $\Theta=0$. Using that a semi-stable bundle is a direct sum of stable bundles one may conclude that

(6.10) If $\mathcal{E}$ is semi-stable and all $c_{i}(\mathcal{E})=0$, then $\mathcal{E}$ has a flat Hermitian metric.

Now a CVHS gives rise to a vector bundle with a flat indefinite Hermitian metric, a situation not directly amenable to elliptic theory. To circumvent this issue, Simpson introduced the above concept of a Higgs bundle and extended these considerations, especially the consequence (6.10). First, a Higgs bundle $(\mathcal{E}, \theta)$ is stable if (6.8) holds for all proper subsheaves $\mathcal{F}$ that are invariant under $\theta$, and similarly for semi-stability. Given a metric on $\mathcal{E}$ one then extends the Higgs operator (6.6) to a connection

$$
\nabla=D^{\prime}+D^{\prime \prime}+\theta+\theta^{*}
$$

where $D^{\prime}+D^{\prime \prime}$ is the metric connection and $\theta^{*}$ is the Hermitian adjoint of $\theta$. The Higgs bundle is said to be HYM if there exists a metric such that the curvature of (6.11) satisfies (6.9). Simpson then extends the arguments of Donaldson and Uhlenbeck-Yau to show that

(6.12) A Higgs bundle $(\mathcal{E}, \theta)$ admits an HYM metric if, and only if, it is a direct sum of stable Higgs bundles.

As before, a corollary is

(6.13) A semi-stable Higgs bundle $(\mathcal{E}, \theta)$ with all $c_{i}(\mathcal{E})=0$ is flat. More precisely, for the HYM metric the connection (6.11) is integrable.

Finally, to complete the story and establish the relation between semi-stable Higgs bundles and CVHS's, Simpson observes that on the set $\mathcal{M}_{\text {Higgs }}$ of equivalence classes of semi-stable Higgs bundles with all $c_{i}(\mathcal{E})=0$ there is a $\mathbb{C}^{*}$-action given by

$$
t(\mathcal{E}, \theta)=(\mathcal{\varepsilon}, t \theta)
$$

It is elementary to see that the fixed points of the action (6.14) are exactly the CVHS's. Indeed, a fixed point is given by an invertible 
endomorphism

$$
T: \mathcal{E} \rightarrow \mathcal{E}
$$

satisfying

$$
T \theta=t \theta T .
$$

The eigenvalues of $T$ are a constant set $\Lambda$ of complex members, and we set (assuming $t$ is not a root of unity)

$$
\left\{\begin{array}{l}
\mathcal{E}_{\lambda}=\operatorname{ker}(T-\lambda I)^{\rho} \\
\Lambda_{p}=\left\{\lambda \in \Lambda: \lambda, t \lambda, \ldots, t^{p-1} \lambda \in \Lambda, t^{p} \lambda \notin \Lambda\right\} \\
\mathcal{E}=\underset{\lambda \in \Lambda_{p}}{\bigoplus} \mathcal{E}_{\lambda} .
\end{array}\right.
$$

Then

$$
\left\{\begin{array}{l}
\mathcal{E}=\oplus \mathcal{E}^{p} \\
\theta: \mathcal{E}^{p} \rightarrow \Omega_{S}^{1}\left(\mathcal{E}^{p-1}\right)
\end{array}\right.
$$

and the conditions for a CVHS are satisfied.

To prove (6.1) one would like to deform the representation of $\pi_{1}(S)$ associated to a Higgs bundle $(\mathcal{E}, \theta)$ into a fixed point under the $\mathbb{C}^{*}$ action (6.14) by taking

$$
\lim _{t \rightarrow 0}(\mathcal{E}, t \theta)
$$

in $\mathcal{M}_{\text {Higgs }}$. As is usual in moduli questions, the issue is that $\mathcal{M}_{\text {Higgs }}$ should be compact. With work this can be proved (cf. [41]), and together with the result that an irreducible representation of $\pi_{1}(S)$ gives rise to a stable Higgs bundle leads to the proof of (6.1).

\section{A recent construction of Nori}

This second Hodge-theoretic construction is based on work in preparation by Nori and is of a quite different character than those discussed above. On the one hand it is formal-homological in the style initiated in Deligne's "Théorie de Hodge", and on the other hand the essential point is geometric and is an extension of the basic Lefschetz picture of building up the homotopy type of an $n$-dimensional smooth variety $X$ by successively attaching cells of dimension $n$ using the locus traced out by the vanishing cycles in a Lefschetz pencil. Nori's work leads to the formulation of a motivic Hodge conjecture (MHC) which would have the following two consequences:

(7.1) Let $X$ be a smooth complex projective variety and $\sigma: \mathbb{C} \rightarrow \mathbb{C}$ an automorphism of the complex numbers with $X^{\sigma}$ denoting the 
corresponding projective variety. Let $Z \in Z^{p}(X)$ satisfy

$$
\psi_{0}(Z)=0, \quad \psi_{1}(Z)=0 .
$$

Then the same is true for $Z^{\sigma} \in Z^{p}\left(X^{\sigma}\right)$.

In particular, although $A J_{X}$ is defined transcendentally, its kernel is invariant under Galois conjugation over the field of definition of $X$.

(7.2) Let $X, Y$ be complex varieties such that for some $q$ there is an isomorphism of MHS's

$$
H^{q}(X) \cong H^{q}(Y) \text {. }
$$

Then the same is true for $X^{\sigma}$ and $Y^{\sigma}$.

Nori's formulations of the MHC and of the related Hodge conjecture for admissable pairs (HCAP's) are based on his construction of the category $\mathcal{A}$ of effective cohomological motives (ECM's). The central geometric point is the following

Definition: A good pair is a pair $Y \subset X$ of affine varieties such that

$$
H^{q}(X, Y)=0, \quad q \neq \operatorname{dim} X .
$$

For example, if $\bar{Y} \subset \bar{X}$ are smooth projective varieties where $\bar{Y}$ is a hyperplane section and $Y \subset X$ is obtained from $\bar{Y} \subset \bar{X}$ by taking out a transverse hyperplane section, then the central point of Lefschetz theory [26] is that $Y \subset X$ is a good pair. The existence of many good pairs is due to Beilinson who proved:

If $X$ is affine and $Y \subset X$ is a closed subvariety with $\operatorname{dim} Y<\operatorname{dim} X$, then there exists $Z$ with $Y \subset Z \subset X$ where $Z$ is a closed subvariety and $Z \subset X$ is a good pair.

Based on this, for an affine variety $X$ there are many ascending chains

$$
Z_{0} \subset Z_{1} \subset \cdots \subset Z_{n}=X \quad(n=\operatorname{dim} X)
$$

of good pairs, and for any such

$$
H^{0}\left(Z_{0}\right) \rightarrow H^{1}\left(Z_{1}, Z_{0}\right) \rightarrow \cdots \rightarrow H^{n}\left(Z_{n}, Z_{n-1}\right)
$$

is a complex that computes $H^{*}(X)$. Note that

(i) (7.4) is a complex of MHS's; and 
(ii) (7.4) is quasi-isomorphic to the singular chain complex of $X$. Thus, in a suitable derived category the Hodge theory (i) and geometric topology (ii) meet at a fundamental level.

Note that if $Y \subset X$ is a good pair, then by étale cohomology the same is true of $Y^{\sigma} \subset X^{\sigma}$. Also note that $\mathcal{U}=X \backslash Y$ is a smooth Zariski open in $X$.

I will now briefly recount Nori's formulation of the HCAP, the MHC and the construction of the category $\mathcal{A}$ of ECM's. Let $X$ be smooth, quasi-projective and $\cup_{i=1}^{m} D_{i}=D \subset X$ a divisor with normal crossings. For $I \subset\{1, \ldots, m\}$ set $D(I)=\cap_{i \in I} D_{i}$ and

$$
Z^{p}(D(I))_{q}^{\prime}=\left\{\begin{array}{c}
\text { free group on all codimenion } p \\
\text { subvarieties } W \subset D(I) \times \Delta^{q} \\
\text { which satisfy Bloch's conditions }
\end{array}\right\}
$$

here Bloch's conditions refer to $W$ meeting the faces of

$$
\Delta^{q}=\left\{\left(x_{0}, x_{1}, \ldots, x_{q}\right): \sum_{i=0}^{q} x_{i}=1\right\}
$$

in subvarieties of the right codimension. This yields a double complex

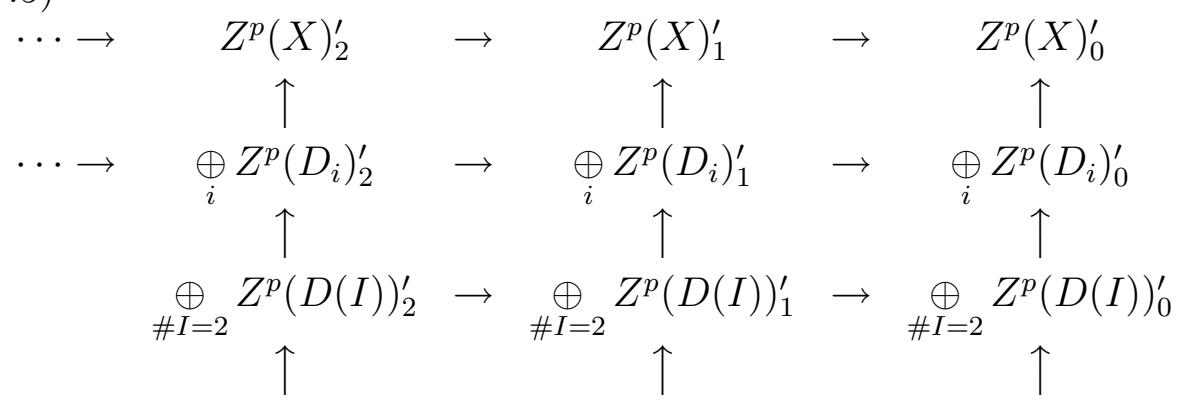

Denoting the total complex by $Z^{p}(X, D)$, Nori formulates the

$H C A P$ : Assume $H^{i}(X, D)=0$ for $i<2 p$. Then

$$
H_{0}\left(Z^{p}(X, D)\right)_{\mathbb{Q}} \rightarrow H g^{2 p}(X, D)_{\mathbb{Q}}
$$

is surjective

Nori observes that even showing that the map (6.6) is defined requires proof.

To explain the MHC, we recall that there is expected to be an abelian category $\mathcal{A}$ of motives with the properties (here $k$ is an algebraically closed field of characteristic zero) 
(A) There is a functor

$$
\begin{array}{ccc}
\text { quasi-projective varieties } / k & \rightarrow & D^{b}(\mathcal{A}) \\
\cup & & \cup \\
X & \rightarrow & |X|
\end{array}
$$

where $D^{b}(\mathcal{A})$ is the derived category of $\mathcal{A}$;

(B) Standard cycle conjecture (SCC). There is a quasi-isomorphism

$$
Z^{p}(X) \bullet, \mathbb{Q} \rightarrow \operatorname{Hom}(\mathbb{Z}(-p)[-2 p],|X|)_{\mathbb{Q}} ;
$$

or more generally a quasi-isomorphism

$$
Z^{p}(X, D) \bullet, \mathbb{Q} \rightarrow \operatorname{Hom}(\mathbb{Z}(-p)[-2 p],|(X, D)|)_{\mathbb{Q}}
$$

(C) Motivic Hodge conjecture (MHC). There is an absolutely fully faithful functor

$$
\mathcal{A} \rightarrow \text { MHS . }
$$

Standard arguments, using the hard Lefschetz theorem, show that

$$
\begin{aligned}
& \mathrm{SCC}+\mathrm{MHC} \Rightarrow \mathrm{HC} \\
& \mathrm{SCC}+\mathrm{MHC} \Rightarrow(6.6) \text { is an isormorphism. }
\end{aligned}
$$

Since $\operatorname{Ext}_{\mathrm{MHS}}^{2}(*, *)=0$, denoting by $F^{m} C H^{p}(X)_{\mathbb{Q}}$ the filtration induced by (6.5) on Chow groups, by (C)

$$
\begin{aligned}
F^{2} C H^{p}(X)_{\mathbb{Q}} & =\operatorname{kernel}\left\{C H^{p}(X)_{\mathbb{Q}} \rightarrow H^{0}\left(\mathbb{H}_{\mathrm{MHS}}(\mathbb{Q}(-p)[-2 p],|X|)\right\}\right. \\
& =\operatorname{kernel} \psi_{0} \cap \operatorname{kernel} \psi_{1}
\end{aligned}
$$

is independent of the embedding $k \hookrightarrow \mathbb{C}$.

We conclude with a few comments on Nori's proposed candidate ECM for $\mathcal{A}$ and the map in (A) above. The category ECM is constructed out of the rings

$$
\text { End } H^{*} \mid F=\left\{a \in \prod_{(X, Y) \in F} H^{*}(X, Y): \text { "conditions" }\right\}
$$

where $F$ is a finite collection of good pairs $Y \subset X$ and the "conditions" refer to the commutativity of certain diagrams

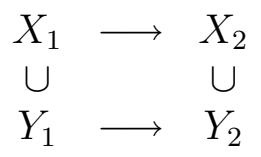


among the good pairs in $F$. The objects are left modules $M$ over End $H^{*} \mid F$ for $F$ a finite set of good pairs. The map

$$
\mathrm{ECM} \rightarrow \mathrm{MHS}
$$

arises from the map

$$
(A=\text { abelian group, } P=\mathrm{MHS}) \rightarrow \operatorname{Hom}(A, P)=\mathrm{MHS},
$$

which gives that

$$
\begin{aligned}
& \operatorname{Hom}\left(H^{*}(X, Y), H_{\mathrm{MHS}}^{*}(X, Y)\right) \text { is a MHS on the abelian } \\
& \text { group End } H^{*}(X, Y) \text {. }
\end{aligned}
$$

The crucial issue, that being the construction of the map in (A) above, proceeds as follows: For $X$ affine the central geometric point is that the map

$$
X \rightarrow\left\{\begin{array}{c}
\text { set of complexes }(6.18) \text { arising } \\
\text { from ascending chains of } \\
\text { good pairs as above }
\end{array}\right\}
$$

leads to a map

$$
\text { affine varieties defined } / k \rightarrow D^{b}(\mathrm{ECM})
$$

into the derived category of ECM. Denoting the right-hand side of (7.7) by $|X|$ and taking $\mathcal{A}=\mathrm{ECM}$ gives (A) for affine $X$ 's. In general, one uses the affine case as the basic building blocks and couples these with more or less standard simplical constructions.

\section{REFERENCES}

[1] M. Atiyah and F. Hirzebruch, Analytic cycles on complex manifolds, Topology 1 (1962), 25-45.

[2] A. Beilinson, J. Bernstein, and P. Deligne, Faisceaux pevers, Asterisque 100 (1981).

[3] J. Bertin and C. Peters, Variations de structures de Hodge, variétés de CalabiYau et symétrie miroir, in Introduction A La Théorie de Hodge 3, Soc. Math. France, 1996.

[4] S. Bloch, On the tangent space to Quillen K-theory, L. N. M. 341 (1974), Springer-Verlag.

[5] _ Lectures on algebraic cycles, Duke Univ. Math. Ser. IV (1980).

[6] J.-L. Brylinski and S. Zucker, An overview of recent advances in Hodge theory, in Several Complex Variables VI, Encyclopedia of Mathematical Sciences 69 (1990), 39-142, Springer-Verlag.

[7] J. Carlson, S. Müller-Stock and C. Peters, Period mappings and period domains, Cambridge Univ. Press 85 (2003). 
[8] E. Cattani, A. Kaplan, and W. Schmid, Degeneration of Hodge structures, Ann. of Math. 123 (1986), 457-535.

[9] $\_, L^{2}$ and intersection cohomologies for a polarizable variation of Hodge structure, Invent. Math. 87 (1987), 217-252.

[10] H. Clemens, Degeneration of Kähler manifolds, Duke Math. J. 44 (1977), 215290.

[11] _ The Neron model for families of intermediate Jacobians acquiring "algebraic" singularities, Publ. Math. I.H.E.S. 58 (1983), 5-18.

[12] - Homological equivalence, modulo algebraic equivalence, is not finitely generated, Publ. Math. I.H.E.S. 58 (1983), 19-38.

[13] A. Collino, Griffiths' infinitesimal invariant and higher $K$-theory on hyperelliptic Jacobians, J. Alg. Geom. 6 (1997), 393-415.

[14] D. Eisenbud, Commutative algebra (with a view toward algebraic geometry), Springer-Verlag 150 (1994).

[15] F. El. Zein and S. Zucker, Extendability of normal functions associated to algebraic cycles, Topics in Transcendental Algebraic Geometry, Ann. of Math. Studies 106 (1984), 269-288, Princeton Univ. Press, Princeton, N.J.

[16] H. Garland, A finiteness theorem for $K_{2}$ of a number field, Ann. of Math. 94 (1971), 534-548.

[17] M. Green, Griffiths' infinitesimal invariant and the Abel-Jacobi map, J. Diff. Geom. 29 (1989), 545-555.

[18] _ Infinitesimal Methods in Hodge Theory, Algebraic Cycles and Hodge Theory, Lecture Notes in Math. 1594 (1993), 1-92, Springer-Verlag, Torino.

[19] M. Green and P. Griffiths, On the tangent space to the space of algebraic cycles on a smooth algebraic variety, to appear in Annals of Math. Studies, Princeton Univ. Press.

[20] $\ldots$, Hodge theoretic invariants for algebraic cycles, IMRN 9 (2003), 477510.

[21] P. Griffiths, A theorem concerning the differential equations satisfied by normal functions associated to algebraic cycles, Amer. J. Math. 101 (1979), 94-131.

[22] P. Griffiths, Topics in Transcendental Algebraic Geometry, Ann. of Math. Studies 106 (1984), Princeton Univ. Press, Princeton, NJ.

[23] M. Kashiwara, The asymptotic behavior of a variation of polarized Hodge structure, Publ. R.I.M.S. Kyoto Univ. 21 (1985), 853-875.

[24] M. Kashiwara and T. Kawai, The Poincaré lemma for a variation of polarized Hodge structure, Proc. Japan Acad. 61 (1985), 164-167.

[25] K. Kato and S. Usui, Classifying spaces of degenerating polarized Hodge structures.

[26] S. Lefschetz, L’analysis et la géometrie algébrique, Paris, Gauthiers-Villars (1924).

[27] J. Le Potier, Fibrés de Higgs et systémes locaux, Séminaire Bourbaki 737 (1991), 221-268.

[28] R. MacPherson and M. Garesky, Intersection homology II, Invent. Math. 79 (1983), 77-.

[29] S. Muller-Stach, Constructing indecomposable motivic cohomology classes on algebraic surfaces, J. Alg. Geom. 6 (1997), 513-543.

[30] D. Mumford, Rational equivalence of zero cycles on surfaces, J. Math. Kyoto Univ. 9 (1969), 195-209. 
[31] J. P. Murre, Algebraic cycles and algebraic aspects of cohomology and $K$ theory, Algebraic Cycles and Hodge Theory, Lecture Notes in Math. 1594 (1993), 93-152, Springer-Verlag, Torino.

[32] J. Nagel, Effective bounds for Hodge-theoretic connectivity, J. Alg. Geom. 11 (2002), 1-32.

[33] T. F. Ng, Regular differential forms on singular varieties, in progress.

[34] M. Nori, Algebraic cycles and Hodge theoretic connectivity, Inv. Math. 111 (1993), 349-373.

[35] _ Notes from a lecture given at Duke University, March 14, 2003.

[36] D. Ramakrishnan, Regulators, algebraic cycles and values of $L$-functions, Contemp. Math. 83 (1989), 183-310.

[37] M. Saito, Mixed Hodge modules, Publ. RIMS, Kyoto Univ. 26 (1990), 221-333.

[38] _ Admissible normal functions, J. Alg. Geom. 5 (1996), 235-276.

[39] S. Saito, Infinitesimal logarithmic Torelli problem for degenerating hypersurfaces in $\mathbb{P}^{n}$, Algebraic Geometry 2000, Adv. Stud. Pure Math. 36 (2000), 401434, Math. Soc. japan.

[40] C. Simpson, Higgs bundles and local systems, Publ. Math. IHES 75 (1992), $5-95$.

[41] Moduli of representations of the fundamental group of a smooth projective variety, I, II, Publ. Math. IHES 77 (1994), 47-129 and 80 (1995), 5-79.

[42] G. Tian, Smoothness of the universal deformation space of compact Calabi-Yau manifolds and its Petersson-Weil metric, in Mathematical Methods in Theoretical Physics, S.-T Yau (editor) (1986), World Scientific, Hong-Kong.

[43] E. Viehweg and K. Zuo, On the Brody hyperbolicity of moduli spaces for canonically polarized manifolds, Duke Math. J. 118 (2003), 103-150.

[44] C. Voisin, Une remarque sur l'invariant infinitesimal des functions normal, $C$. R. Acad. Sci. Paris Sér I 307 (1988), 157-160.

[45] _ Transcendental Methods in the Study of Algebraic Cycles, Algebraic Cycles and Hodge Theory, Lecture Notes in Math. 1594 (1993), 153-222, Springer-Verlag, Torino.

[46] _ Sur l'application d'Abel-Jacobi des variétés de Calabi-Yau de dimension trios, Ann. Sci. École Norm. Sup. 27 (1994), 129-172.

[47] Hodge Theory and Complex Algebraic Geometry I, II, Cambridge Studies in Advanced Math. 76 (2002), Cambridge Univ. Press.

[48] _ A counterexample to the Hodge conjecture extended to Kähler varieties, IMRN 20 (2002), 1057-1075.

[49] A A generic Torelli theorem for the quintic threefold, London Math. Soc. Lecture Note Ser. 264 (1999), Cambridge.

[50] _ The Griffiths group of a general Calabi-Yau threefold is not finitely generated, Duke Math. J. 102, 151-186.

[51] K. Zuo, On the negativity of kernels of Kodaira-Spencer maps on Hodge bundles and applications, Asian J. Math. 4 (2002), 279-301.

Institute For AdVAnced Study 